\title{
Gravity-mediated dark matter annihilation in the Randall-Sundrum model
}

\author{
T.D. Rueter, ${ }^{a, b}$ T.G. Rizzo ${ }^{b}$ and J.L. Hewett ${ }^{b}$ \\ ${ }^{a}$ Department of Physics, Stanford University, \\ 382 Via Pueblo, Stanford, CA 94305, U.S.A. \\ ${ }^{b} S L A C$ National Accelerator Laboratory, Stanford University, \\ 2575 Sand Hill Road, Menlo Park, CA 94025, U.S.A. \\ E-mail: tdr38@stanford.edu, rizzo@slac.stanford.edu, \\ hewett@slac.stanford.edu
}

ABSTRACT: Observational evidence for dark matter stems from its gravitational interactions, and as of yet there has been no evidence for dark matter interacting via other means. We examine models where dark matter interactions are purely gravitational in a RandallSundrum background. In particular, the Kaluza-Klein tower of gravitons which result from the warped fifth dimension can provide viable annihilation channels into Standard Model final states, and we find that we can achieve values of the annihilation cross section, $\langle\sigma v\rangle$, which are consistent with the observed relic abundance in the case of spin-1 dark matter. We examine constraints on these models employing both the current photon line and continuum indirect dark matter searches, and assess the prospects of hunting for the signals of such models in future direct and indirect detection experiments.

KEYWORDS: Phenomenology of Large extra dimensions

ARXIV EPRINT: 1706.07540 


\section{Contents}

1 Introduction 1

2 Randall-Sundrum model framework 3

3 Analysis $\quad 9$

3.1 Thermal relic abundance calculation $\quad 9$

$\begin{array}{lll}3.2 & \text { Dark matter indirect detection } & 15\end{array}$

3.3 Dark matter spin-independent direct detection 22

4 Discussion and conclusions $\quad 23$

A Cross sections and width for graviton-mediated annihilation: graviton width

B Dark matter annihilation cross sections $\quad 25$

B.1 Scalar dark matter 25

B.2 Fermionic dark matter 27

$\begin{array}{lll}\text { B.3 Vector dark matter } & 27\end{array}$

\section{Introduction}

Observations indicate that roughly $85 \%$ of the matter in the universe is non-luminous, with this conclusion being drawn from the apparent gravitational effects of cold dark matter particles. The nature of this dark matter (DM), and its potential interactions with the Standard Model (SM), remain mysterious. A widely studied DM candidate is the thermal relic weakly interacting massive particle (WIMP) scenario, where the observed relic density implies that the DM has a mass in the GeV-TeV range and possibly couples with weak interaction strength (or less) to the SM fields directly or through an additional mediator field $[1,2]$. If the DM is a SM singlet field, a mediator is a necessary ingredient in order to generate non-gravitational signatures, and the DM itself may only be a small part of a larger Dark Sector. Despite numerous attempts, a non-gravitational DM signal has yet to be observed and the WIMP-like scenario is already reasonably constrained by both direct $[3,4]$ and indirect $[5-12]$ searches, as well as by complimentary DM and mediator searches at low energy accelerators [13] and the LHC. ${ }^{1}$

In this paper, we examine DM in theories with additional spatial dimensions where the DM itself has purely gravitational interactions. We work within the framework of

\footnotetext{
${ }^{1}$ See for example refs. [14-19].
} 
the warped extra dimension scenario of Randall and Sundrum (RS) [20] where DM communication with the SM may then occur via a Kaluza-Klein graviton mediator present in the model. The RS model is an attractive scenario, presenting well-known solutions to the gauge hierarchy and the fermion mass hierarchy problems. In this framework the gauge hierarchy is generated via an exponential warp-factor appearing in the 5-dimensional metric, with the Higgs field, together with its vev, being located on or near the so-called $\mathrm{TeV}$-scale brane. Mass scales are thus reduced by this exponential factor in comparison to the Planck scale. In addition, the suitable placement of the SM gauge and fermion fields in the 5-dimensional bulk [21,22], allows for the reduction of the fermion mass hierarchy to that of selecting an appropriate set of $\mathcal{O}(1)$ numbers. Of course this RS picture is not without its own problems: it faces some rather severe constraints from $(i)$ precision electroweak measurements [23-25], (ii) the existence of tree-level Flavor-Changing Neutral Currents [26, 27], as well as (iii) direct searches for the predicted Kaluza-Klein (KK) excitations of the graviton and SM gauge fields at the LHC [14-19]. Together, these constraints generally force the $\mathrm{KK}$ scale near the $\sim 10 \mathrm{TeV}$ range even when there is a custodial gauge symmetry present $[28]^{2}$

The RS scenario naturally realizes the interesting possibility that the DM communicates with the SM via purely gravitational interactions, the only interactions that we know for sure that DM possesses, utilizing the KK graviton excitations as mediators between the DM and the SM. This concept was pioneered in [30-32] and has been adapted for use in the form of a Simplified Model in [33] along the lines suggested by the LHC Dark Matter Working Group [34, 35]. Here, we explore this enticing possibility in more detail by employing a number of specific benchmark scenarios which make full use of the RS model building features of varying localizations for the SM gauge and fermion fields, brane localized kinetic terms [36], as well as differing graviton KK mass scales. In particular, $(i)$ we provide an exact treatment of the graviton exchange process leading to the velocity weighted thermal DM annihilation cross section, without employing a velocity expansion. As we will see below, the velocity expansion approximation is questionable near KK resonances and such regions are found to be of particular importance in our analysis. (ii) We consider in detail the various possibilities of the DM spin, spin-0,1/2 and 1 , and determine the DM velocity dependence for all potential SM annihilation channels. We demonstrate that the success of this scenario requires the DM to be spin- 1 and have a mass $\gtrsim 350 \mathrm{GeV}$ to avoid velocity suppression of the annihilation cross section. (iii) We include the contributions of multiple graviton KK excitations; this is found to be important since the behavior of the thermal cross section near these additional peaks opens up new successful parameter space regions. However, we observe that the relative behavior of the KK resonance regions vary in detail between the different benchmark scenarios. (iv) We examine the direction and indirect detection constraints in detail. As we will show below, indirect detection searches for both antiprotons and gamma rays are found to potentially constrain interesting parameter space regions, while there is no observable signature in direct detection experiments.

\footnotetext{
${ }^{2}$ See, however, [29] for a possible way to evade these significant issues and reduce the KK gauge boson masses to $\sim 1 \mathrm{TeV}$.
} 
The organization of this paper is as follows: after our Introduction in the present section, we provide an overview of the basics of the RS framework in section 2 where our benchmark models are also presented. Section 3 contains our analysis of the thermal relic abundance via graviton KK exchange for DM particles of various spins, as well as a discussion of the constraints that arise from direct and indirect DM searches. A discussion and our conclusions can be found in section 4 . The appendix summarizes the formulae for the KK graviton partial widths, as well as the differential cross sections for DM annihilation of various spins.

\section{Randall-Sundrum model framework}

In this section we discuss the general model framework that we follow in the analysis below. The RS scenario is based on a slice of $\mathrm{AdS}_{5}$ spacetime bounded by two 4-dimensional Minkowski branes. The fifth dimension, denoted by $y=r_{c} \phi$, is bounded at either end by the UV-, or Planck, brane located at $y=0$ and the IR-, or TeV, brane sitting at $y=\pi r_{c}$. The associated metric is $d s^{2}=e^{-2 k y} \eta_{\mu \nu} d x^{\mu} d x^{\nu}-d y^{2}$. The exponential represents the warp factor (which generates the gauge hierarchy), while the parameter $k$ governs the degree of curvature of the $\mathrm{AdS}_{5}$ space. The relation $\bar{M}_{P l}^{2}=M_{5}^{3} / k$ is thus derived from the 5 -D action. The scale of physical phenomena on the IR-brane is given by $\Lambda_{\pi} \equiv \bar{M}_{P l} e^{-k r_{c} \pi}$ with $\Lambda_{\pi} \sim$ few TeV implying $k r_{c} \sim 11-12$. In the simplest picture, the SM fields reside on the IR-brane with only gravity propagating in the $5^{\text {th }}$ dimension. The mass of the $n^{\text {th }}$ KK graviton excitation is $m_{n}^{G}=x_{n}^{G} k \Lambda_{\pi} / \bar{M}_{P l}$, with $x_{n}^{G}$ being the roots of the $J_{1}$ Bessel function. The coupling strength of the graviton KK states to the SM fields is $\Lambda_{\pi}^{-1}$ [37-41].

The small size of the additional dimension allows for the SM fields to propagate in the bulk. This scenario has attractive model-building features such as the potential to explain the fermion mass hierarchy. In this case the gauge bosons also have KK excitations with their masses being governed by the roots of the equation

$$
J_{1}\left(x_{n}^{A}\right)+x_{n}^{A} J_{1}^{\prime}\left(x_{n}^{A}\right)+\alpha_{n}\left[Y_{1}\left(x_{n}^{A}\right)+x_{n}^{A} Y_{1}^{\prime}\left(x_{n}^{A}\right)\right]=0,
$$

with the numerical coefficients $\alpha_{n}$ being determined by the boundary conditions on the UV-brane. The gauge KK masses are then $m_{n}^{A}=x_{n}^{A} k \Lambda_{\pi} / \bar{M}_{P l}$. When the fermions also reside in the bulk, additional parameters $\left(m_{i}=k \nu_{i}\right.$ or $\left.=-k c_{i}\right)$ are introduced corresponding to the bulk fermion masses. These parameters determine the localization of the fermion wavefunction. As the Higgs boson is kept on or near the IR-brane (to address the gauge hierarchy), a consistent framework emerges if light flavor fermions are localized near the UVbrane and the third generation quarks are localized on or near the IR-brane. The addition of brane localized kinetic terms (BLKTs), which arise naturally from quantum effects [36], modify the spectrum and couplings of the graviton, gauge, and fermion KK states [37-41].

To be specific, we consider five benchmark points in the RS parameter space that differ in the localizations of the various SM and DM particles and in the masses of the lowest lying KK gravitons. For all of these benchmarks the Higgs field and the associated Goldstone bosons, i.e., essentially the longitudinal components of the massive gauge bosons that result from spontaneous symmetry breaking, reside on the IR-brane. To be general, 
we will in some cases, also allow for the presence of brane localized kinetic terms (BLKTs) for the various bulk fields so that, in the notation of refs. [37-41], the 5-D gravitational action will be given by

$$
S_{G}=\frac{M_{5}^{3}}{4} \int d^{4} x \int r_{c} d \phi \sqrt{-G}\left\{R^{(5)}+\left[2 \gamma_{0} / k r_{c} \delta(\phi)+2 \gamma_{\pi} / k r_{c} \delta(\phi-\pi)\right] R^{(4)}+\ldots\right\},
$$

where $\gamma_{0, \pi}$ are the corresponding dimensionless UV- and IR-brane terms, respectively. Similarly, for the SM and possible DM gauge fields we have

$$
S_{V}=\frac{-1}{4} \int d^{4} x \int r_{c} d \phi \sqrt{-G}\left\{F_{A B} F^{A B}+\left[2 \delta_{0} / k r_{c} \delta(\phi) 2 \delta_{\pi} / k r_{c} \delta(\phi-\pi)\right] F_{\mu \nu} F^{\mu \nu}+\ldots\right\},
$$

where $\delta_{0, \pi}$ are the UV- and IR-brane terms. We assume for simplicity that the gauge fields corresponding to the three SM gauge groups all have common BLKT values $\delta_{0}$ and $\delta_{\pi}$, and generically $\delta_{0} \neq \delta_{\pi}$. If the $\mathrm{DM}$ is identified as a spin- 1 gauge bulk field, this assumption will apply to the DM field as well. For the various fermions in the bulk [23-25] we have similarly

$$
\begin{aligned}
S_{F}=\int d^{4} x \int r_{c} d \phi \sqrt{-G}\{ & \frac{-i}{2} \bar{\Psi} \Gamma^{A} \partial_{A} \Psi+\left[2 \tau_{0} / k r_{c} \delta(\phi)\right. \\
& \left.\left.+2 \tau_{\pi} / k r_{c} \delta(\phi-\pi)\right] \frac{-i}{2} \bar{\Psi} \gamma^{\mu} \partial_{\mu} \Psi+\text { h.c. }-\operatorname{sgn}(\phi) m_{f_{\Psi}} \bar{\Psi} \Psi\right\},
\end{aligned}
$$

where $\tau_{0, \pi}$ are the corresponding UV- and IR-brane terms which we again assume take on identical values for all SM fermion representations for simplicity. As usual, each of the various fermion representations take on distinct discrete values for the bulk mass parameter, $m_{f}=k \nu_{f}$, which will peak their wavefunctions towards either the IR- or UV- branes. These values are predominantly determined by replicating the fermion mass hierarchy.

In the original RS model where all the SM fields reside on the $\mathrm{TeV}$ brane, and in the absence of graviton BLKTs, the SM fields couple to the full set of graviton KK excitations with universal strength, $\Lambda_{\pi}^{-1}$. Here we scale the couplings of each field to this quantity, so the effect of both the field localizations and the various BLKTs can be easily tracked. For example, the existence of graviton BLKTs rescales the KK graviton tower couplings to the fields localized on the $\mathrm{TeV}$ brane by factors of (here the index $n$ labels the state in the graviton KK tower)

$$
\lambda_{n} \equiv\left[\frac{1+2 \gamma_{0}}{1+\left(x_{n}^{G} \gamma_{\pi}\right)^{2}-2 \gamma_{\pi}}\right]^{1 / 2} .
$$

This 'correction' factor clearly takes on a value of unity in the absence of graviton BLKTs. Recall that the graviton KK masses themselves are also influenced by the presence of BLKTs and are given by $m_{n}^{G}=x_{n}^{G} k \epsilon=x_{n}^{G} k \Lambda_{\pi} / \bar{M}_{P l}$, with $x_{n}^{G}$ being the roots of the transcendental equation

$$
J_{1}\left(x_{n}^{G}\right)-\gamma_{\pi} x_{n}^{G} J_{2}\left(x_{n}^{G}\right)=0,
$$

where $J_{1,2}$ are the usual Bessel functions of the first kind.

For the case of bulk gauge fields, the graviton couplings to the 5-D Yang-Mills kinetic term in the presence of BLKTs are further rescaled by additional factors of

$$
\delta_{n}=\frac{2\left(1-J_{0}\left(x_{n}^{G}\right)\right)+\left(\delta_{\pi}-\gamma_{\pi}\right)\left(x_{n}^{G}\right)^{2} J_{2}\left(x_{n}^{G}\right)}{\left.\left(\pi k r_{c}+\delta_{\pi}+\delta_{0}\right)\left(x_{n}^{G}\right)^{2} \mid J_{2}\left(x_{n}^{G}\right)\right) \mid} .
$$


The graviton KK coupling strengths, in units of $\Lambda_{\pi}^{-1}$, are given by the product $\lambda_{n} \delta_{n}$. We briefly note that while the graviton KK states couple to the 5-D Yang-Mills kinetic Lagrangian for bulk gauge fields, this is sometimes referred to in the literature as a coupling to the transverse polarizations of the gauge fields in the bulk. This is certainly the case for massless gauge bosons, but for massive gauge bosons where the spontaneous symmetry breaking occurs on the IR-brane, the kinetic part of the coupling of the longitudinal polarization to the $n^{\text {th }}$ graviton KK mode also arises from the 5-D Yang-Mills kinetic term, and thus is rescaled by a factor of $\delta_{n}$. We emphasize that the Higgs-gauge interactions on the IR-brane, including spontaneous symmetry breaking, are not rescaled by the $\delta_{n}$ factor.

Correspondingly, for the case where the SM fermions, $f_{L, R}$, reside in the bulk, in addition to the overall factor of $\lambda_{n}$ above, their couplings to the graviton KK tower fields are further rescaled by factors of $c_{L, R n}^{f}$. These are the coupling strengths of left-handed and right-handed bulk fermions $f$ to the $n^{\text {th }}$ graviton KK state, and are determined through numerical integration over the appropriate 5 -D wavefunctions. Clearly, the factors $c_{L, R n}^{f}$ will be $\nu_{f_{L, R}}$-dependent. The set of Feynman rules that are required to compute the DM annihilation cross section are given explicitly in the next section.

We now define our five benchmark points. These points have been chosen in order to provide distinct examples of the various RS model features in order to illustrate the potential variation in the results. The first 3 benchmarks are rather simple: in these cases we assume that all of the SM fields, as well as the DM, are constrained to lie on the IRbrane; we denote these benchmark points as Brane models. These points differ only in the assumed value of the mass of the lightest graviton KK state in each case, which we take to be 750,1500 or $3000 \mathrm{GeV}$, respectively. Since in these cases the only free parameter in the model is $\Lambda_{\pi}$, we must choose this parameter such that we are not in conflict with the $13 \mathrm{TeV}$ LHC searches for graviton production and decay in all channels, with the most relevant being: $G_{1} \rightarrow j j, \gamma \gamma$ and $\ell^{+} \ell^{-}[14-19] .^{3}$ Figure 1 shows the production cross sections for the first graviton KK state at the $13 \mathrm{TeV}$ LHC for the relevant diphoton and dilepton final state from which the $\Lambda_{\pi}$ limits can be extracted. The dijet mode is always found to yield an inferior constraint, since for SM fields on the IR-brane one finds $\sigma_{j j} / \sigma_{\gamma \gamma} \simeq 14$, which is not enough to overcome the much larger SM background for dijet production. To lie safely below the current constraints for the lightest KK graviton masses assumed above, we choose $\Lambda_{\pi}=135(90,30) \mathrm{TeV}$, respectively, for these 3 benchmark models. Note that these benchmark points do not address the gauge hierarchy.

The other two benchmark models are more complex. In the first case (hereafter referred to as MOR), the details of the model are provided in ref. [42]. In this case the third generation quarks are confined to the IR-brane while all other SM fermions are localized sufficiently close to the UV-brane so that their couplings to the graviton KK modes can be safely ignored. Gauge fields are placed in the bulk. Here one finds, e.g., that $\lambda_{1} \simeq 0.125, \delta_{1}=0.5$ with $\Lambda_{\pi}=6 \mathrm{TeV}$, which is chosen to satisfy the LHC search constraints above given an assumed mass of the lightest KK graviton of $750 \mathrm{GeV}$. The values of the BLKT parameters are chosen to be $\gamma_{\pi}=-7.652, \delta_{0, \pi}=-10$ and $1+2 \gamma_{0}=25$. Given these inputs the values of

\footnotetext{
${ }^{3}$ Of course we will ensure that these constraints are also satisfied for the other benchmark models as well.
} 


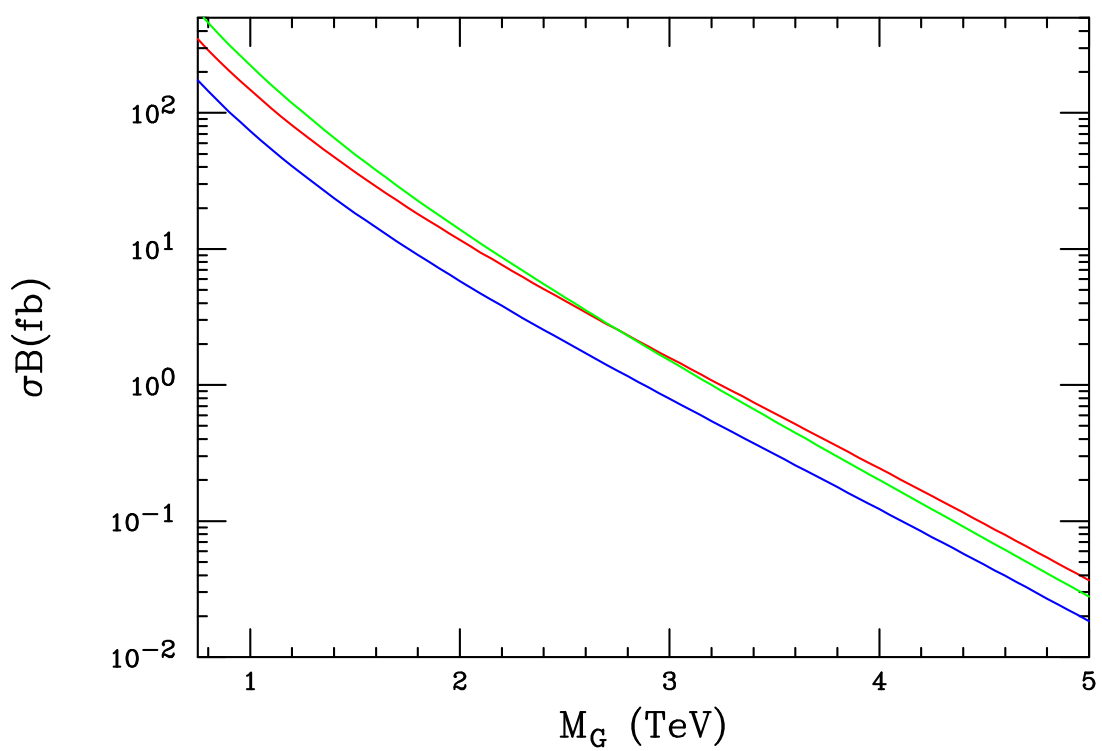

Figure 1. Production cross sections for the lightest KK graviton production at the $13 \mathrm{TeV}$ LHC for several of our benchmark models. For the cases where the SM is constrained to the TeV brane the red(blue) curve is weighted by the branching fraction into the $\gamma \gamma\left(\ell^{+} \ell^{-}\right)$final states with production occurring through both $g g$ and $q \bar{q}$ annihilation. In the GW benchmark case (green), the result includes the branching fraction into diphotons and only has contributions from the $g g$ production channel since there is essentially no coupling to light quarks (or leptons) in this case. For the MOR benchmark, the result is similar to that for GW apart from an overall normalization factor of $\simeq 0.03$.

$\lambda_{n}, \delta_{n}$ can be easily calculated. Note that the MOR model satisfies the original motivation of RS models in explaining the gauge hierarchy. It was originally motivated by the bygone $750 \mathrm{GeV}$ diphoton excess, but remains in agreement with the data for this value of $\Lambda_{\pi}$.

The final benchmark point (hereafter referred to as GW) is significantly more complex. ${ }^{4}$ In this case all SM fermions are localized at various places in the bulk and the gauge, fermion and graviton BLKTs are assumed to be universal, i.e., $\tau_{\pi}=\gamma_{\pi}=\delta_{\pi}$ for the IR-brane and similarly for the UV BLKTs. $\nu_{t_{R}}=-0.5$ and $\nu_{b_{R}} \simeq-0.6$ are assumed. The values of $\tau_{\pi, 0}$ and the other $\nu_{f}$ parameters are then floated to obtain the observed fermion masses, the correct structure and values for the CKM matrix elements and a sufficiently strong suppression of the couplings of the SM zero-mode fermions to the KK gauge tower fields. The avoidance of FCNCs and the absence of potentially large gauge couplings to the KK graviton tower are also employed as additional constraints. Given these conditions, rather unique ranges for the values of the remaining parameters are obtained in this scenario; the value of $\lambda_{1}^{-1} \Lambda_{\pi}=28 \mathrm{TeV}$ with a lightest $\mathrm{KK}$ graviton mass of $3 \mathrm{TeV}$ will be employed in the numerical study of this benchmark below although other values are possible. For more details, see ref. [29].

We next discuss the properties of the DM field. To be explicit, we will generally allow the DM to be either a real scalar, a neutral, vector-like Dirac fermion, or a new neutral massive gauge field, where in all cases it will be assumed to be a SM singlet. For all

\footnotetext{
${ }^{4}$ For more details of this scenario see ref. [29].
} 


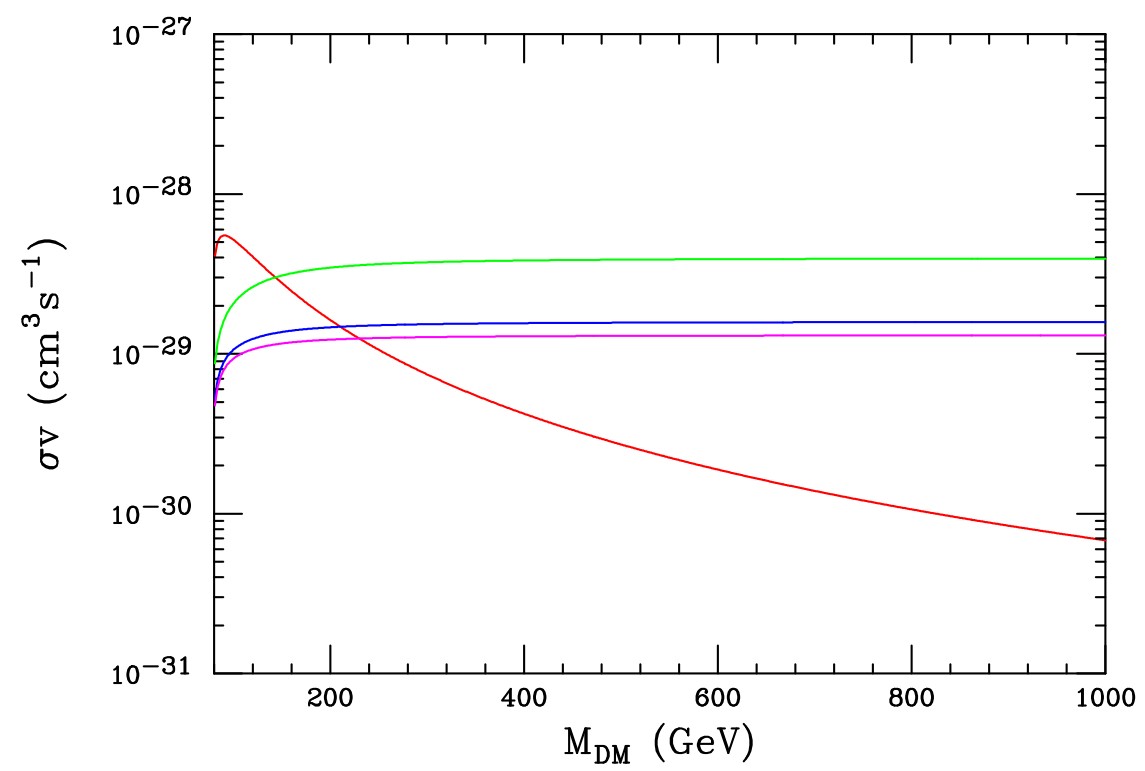

Figure 2. Higgs-mediated pair annihilation cross section scaled by the relative DM velocity as a function of the DM mass; note that this cross section is not thermally averaged. The red curve corresponds to the case of scalar dark matter, $S$, for the specific process $S S \rightarrow h \rightarrow W^{+} W^{-}$, with $\tilde{\lambda}=10^{-2}$ (as discussed in the text). The green (blue, magenta) curves are for the analogous vector DM annihilation channel assuming an effective coupling of $10^{-2}$ as described in the text. Here the heavier scalar mass is assumed to be $3(5,7) m_{D M}$, respectively.

these possibilities we will assume that the DM states annihilate to SM fields solely (or dominantly) via their KK graviton interactions, in line with the premise of this paper. However, this introduces some potential difficulties when building a realistic model, which we now discuss. For example, when we introduce a new real scalar field $S$ as the DM it is well-known that the scalar portal mechanism may be operable [43]. In this case, nothing forbids the $S$ from interacting with the SM fields via an induced trilinear coupling to the Higgs of the form $\sim \tilde{\lambda} v_{H} S h^{2}$ which results from SSB in the SM. For example, if $m_{S}>M_{W}$, the DM annihilation process $S S \rightarrow h^{*} \rightarrow W^{+} W^{-}$can occur in addition to annihilation through the graviton KK channels and may even dominate. However, we find that if $\tilde{\lambda} \lesssim 10^{-2}$, then these SM-mediated processes have sufficiently small rates so that $S$ can never achieve the thermal relic density target via the portal mechanism; this is illustrated in figure 2. We assume this small coupling in the analysis below, and ignore this potential annihilation contribution. For all the benchmark points considered here we take the DM singlet scalar to be localized on the TeV brane, as is the case for the SM Higgs.

In the case of vector $\mathrm{DM}$ (which we assume is a new $\mathrm{U}(1)_{D}$ gauge field $V$ ), the situation is a bit more complex. First, we assume that the mass of this gauge field is generated via its coupling to a real scalar field, $S$, localized on the IR-brane, which gets a TeV-scale vev, $v_{S}$, via the usual Higgs mechanism. Necessarily, we must have $m_{S}>m_{V}$ so that $V$ is the lightest new state and can be identified as the DM. In this case, since $S$ gets a vev, $S$ must mix with the usual SM Higgs field through a phenomenologically small (constrained by 
LHC measurements ${ }^{5}$ ) angle, $\theta$, forming the mass eigenstates $h_{1,2}$. Since a $V V S$ coupling is generated by the non-zero vev $v_{S}$, the DM $V$ can pair annihilate to SM fields via, e.g., the process $V V \rightarrow h_{1,2} \rightarrow W^{+} W^{-}$, which is controlled by the overall effective coupling $\left(g_{D} / g\right) s_{\theta} c_{\theta}$ where $g_{D}[g]$ is the value of the $\mathrm{U}(1)\left[\mathrm{SU}(2)_{L}\right]$ gauge coupling. As shown in figure 2 , taking $\left(g_{D} / g\right) s_{\theta} c_{\theta} \lesssim 10^{-2}$ with various assumed values of the mass of $h_{2}$, we see that this mechanism does not lead to a large enough cross section to generate the observed relic density. We assume that this coupling is sufficiently suppressed in the analysis below, so that we can safely ignore this process in our further discussion. Similar arguments to these can be made in order to avoid a substantial corresponding contribution to DM pair annihilation arising from a gauge portal $[46,47]$. For example, we assumed (i) that there are no states in the model that share both $\mathrm{SM}$ and new $\mathrm{U}(1)_{D}$ couplings at lowest order in the absence of mixing among the matter fields, or that (ii) there exists a $Z_{2}$ symmetry under which $\mathrm{V}$ is odd so that kinetic mixing is absent.

The remaining property to be determined in our benchmark models of gravitationally interacting DM is the localization of the DM itself within the extra dimension. In the first three cases where the SM is IR-brane localized, we localize the DM there as well. In the case of scalar (vector) DM, its coupling to the KK graviton states is then the same as that for the Higgs (SM $Z$ ) boson apart from the value of the DM mass.

When the DM is a fermion, it also is assumed to couple to the KK gravitons in the same way as does any other similarly localized SM fermion, apart from its mass. The DM localization assignments are more complex in both the MOR and GW benchmark scenarios. In the MOR case, for scalar (vector) DM, the $S(V)$ field is localized to the IR-brane as is the SM Higgs. In the case of fermionic DM for the MOR benchmark, the DM field is localized to the $\mathrm{TeV}$ brane and couples in a manner analogous to the third generation of quarks. For the GW model, fermionic DM lies in the bulk and couples in a manner similar to the right-handed top quark. For vector DM in this case, $V$ is assumed to behave in a manner similar to the SM $Z$ boson apart from its mass.

In all cases we treat the DM mass as a free parameter except for the requirement that $m_{D M}<m_{G^{1}}$, which we assume for simplicity, thus avoiding DM annihilation into graviton KK states. ${ }^{6}$ Once above this threshold, the process obtains contributions from DM exchanges in both the $t$ - and $u$-channels, from a 4-pt coupling, as well as via multiple $s$ channel KK graviton exchanges. These $s$-channel terms will clearly dominate in the regions near graviton KK resonances through triple graviton coupling processes such as $G_{3} \rightarrow 2 G_{1} .^{7}$ These types of decays (in the absence of graviton BLKTs) were considered in ref. [48] and the analysis presented there can be straightforwardly generalized to cover this more complex situation. To estimate the size of such contributions, we consider the simplest situation, corresponding to our first three benchmark models, where all SM fields are constrained to lie on the IR-brane. For these benchmarks we find that $B\left(G_{3} \rightarrow 2 G_{1}\right) \simeq 15.2 \%$, indicating that this would be an important, though not dominant, final state and that the overall DM

\footnotetext{
${ }^{5}$ Such models are constrained by e.g. ref. [44, 45].

${ }^{6}$ We will make this assumption for all DM spin assignments in all benchmark cases independently of where the DM is localized.

${ }^{7}$ Recall that the graviton KK spectrum satisfies the ordering $m_{G_{2}}<2 m_{G_{1}}<m_{G_{3}}$.
} 
annihilation cross section in this mass range would not be much influenced by the presence of this contribution. Note that in the mass range $2 m_{D M}<m_{G_{3}}$, phase space arguments suggest that this $2 G_{1}$ final state will be even less important. Thus we conclude that our results would not be significantly altered if our assumption of $m_{D M} \leq m_{G_{1}}$ is violated.

We now turn to a discussion of DM annihilation to the various SM fields for each of these benchmark scenarios.

\section{Analysis}

In this section we describe the computation of the thermal relic abundance, as well as the cross sections for direct and indirect detection for gravitationally mediated DM interactions.

\subsection{Thermal relic abundance calculation}

Before proceeding with our thermal relic calculation, we note that the Randall-Sundrum geometry, when stabilized by a bulk scalar via the Goldberger-Wise mechanism, can give rise to a non-standard cosmology above a critical temperature $T_{c} \lesssim \mathcal{O}(\mathrm{TeV})$. It has been shown using the AdS/CFT correspondence that at high temperatures, the AdS-Schwarzchild geometry with an event horizon replacing the IR-brane is energetically favorable, and there is a first order phase transition to the RS geometry at $T_{c}$ as discussed in ref. [51]. This appears to bring into question the viability of the thermally produced WIMP scenario in the RS background. However, once the phase transition into RS geometry has occurred, tunneling back into the AdS-Schwarzchild phase requires superheating at a temperature much larger than $T_{c}$ [52]. Thus when reheating occurs due to bubble collisions in the phase transition, we need not consider a constraint on the reheating temperature from $T_{c}$, and we can recover a standard cosmological evolution with a thermal relic WIMP.

We also note that we neglect radion exchange in our calculation. Generically the radion is lighter than the first KK graviton, though its mass is not tied to the geometry in the same way as the KK graviton masses are, and it is a much more narrow resonance. This is due to both the radion being lighter as well as the radion's coupling to matter being weaker than that of the graviton by a factor of $1 / \sqrt{3}$ in the notation of refs. [37-41]. As a result of the narrowness of the radion and its decreased coupling to matter, the cross-sections for radion exchange are greatly suppressed away from the radion resonance. Resonant $s$-channel exchange of the radion could potentially saturate the dark matter relic density, but only if the dark matter mass was almost exactly half of the radion mass. Taking $m_{\text {radion }} / m_{G 1} \sim 2 / 3$ and comparing the cross-section for DM annihilating into an SM final state from $s$-channel exchange of an on-resonance radion to an on-resonance graviton, we

find $\sigma_{\text {radion }} / \sigma_{\text {graviton }} \lesssim 1 \%$. The Doppler broadening due to the thermal average serves to further suppress the narrow radion resonance. Since the radion may be lighter than the dark matter, annihilation into two radions could also be a relevant channel, as noted in ref. [31]. However, the $t$ - and $u$-channel diagrams do not benefit from the resonant behavior of the $s$-channel considered earlier, and thus are suppressed. Similarly, DM annihilation into two radions via a resonant $s$-channel radion exchange is not allowed kinematically, and so is also suppressed. Finally, there exists a four point interaction between two radions and 
two dark matter particles, but this channel does not benefit from any resonant enhancement while still having the same number of powers of the coupling as the previous channels, and is correspondingly suppressed. We thus concentrate our efforts on the KK graviton states as mediators of dark matter interaction with the SM.

In the thermal freeze-out scenario, dark matter is produced in thermal equilibrium with the hot, dense early universe, and then freezes out as the universe expands and cools. ${ }^{8}$ In such cases the evolution of the number density of dark matter $n_{\chi}$ is governed by the Boltzmann equation:

$$
\frac{d n_{\chi}}{d t}=-3 H n_{\chi}-\left\langle\sigma v_{M \varnothing l}\right\rangle\left[n_{\chi}^{2}-\left(n_{\chi}^{\mathrm{eq}}\right)^{2}\right]
$$

where $n_{\chi}^{\text {eq }}$ is the number density of DM particles that would exist in thermal equilibrium, $\sigma=\sum_{f \in S M} \sigma(\chi \chi \rightarrow f)$ is the sum of all annihilation channels into SM final states, $H(T)$ is the Hubble expansion rate as function of temperature $\mathrm{T}$, and $v_{M ø l}$ is the Møller velocity as defined in ref. [50]. In the early universe, the DM is in thermal equilibrium and the first term in the equation governs the number density evolution. As the universe expands and cools, the interaction rate $\Gamma_{\chi} \equiv n_{\chi}\left\langle\sigma v_{M ø l}\right\rangle$ falls below $H(T)$ and the dark matter freezes out of thermal equilibrium and its number density in a co-moving frame remains (approximately) constant.

In this framework the thermal relic abundance of the dark matter is determined by the value of $\left\langle\sigma v_{M ø l}\right\rangle$. Here we calculate this quantity analytically and do not use the velocity expansion which can be invalid in the neighborhood of any $s$-channel resonances; such contributions are an important region in our scenario as we will see in our discussion below. The thermal average is formally defined with each annihilating particle in its own thermal bath, and we can write $\left\langle\sigma v_{M ø l}\right\rangle$ as

$$
\left\langle\sigma v_{M \varnothing l}\right\rangle=\frac{\int \sigma v_{M \varnothing l} e^{-E_{1} / T} e^{-E_{2} / T} d^{3} p_{1} d^{3} p_{2}}{\int e^{-E_{1} / T} e^{-E_{2} / T} d^{3} p_{1} d^{3} p_{2}}
$$

where we have assumed $T \lesssim 3 m_{\chi}$, with $m_{\chi}$ being the dark matter mass, so that we may use Maxwell-Boltzmann statistics, following ref. [50]. We have checked numerically that this is always an excellent approximation for the parameter ranges of interest to us here. In particular, the dimensionless ratio $x_{f} \equiv m_{\chi} / T_{f}$, where $T_{f}$ is the freeze-out temperature, typically takes on values of 20-30 [53]. For simplicity in our analysis we set $x_{f}=25$. The above expression can be reduced to a one dimensional integral over the Mandelstam variable $s=\left(p_{1}+p_{2}\right)^{2}$ where the $p_{i}$ are the 4-momenta of the initial DM states, giving

$$
\left\langle\sigma v_{M \varnothing l}\right\rangle=\frac{1}{8 m_{\chi}^{4} T K_{2}^{2}\left(m_{\chi} / T\right)} \int_{4 m_{\chi}^{2}}^{\infty}\left(s-4 m_{\chi}^{2}\right) \sqrt{s} \sigma K_{1}\left(\frac{\sqrt{s}}{T}\right) d s .
$$

Here the annihilation cross section $\sigma$ is a function only of the masses of the various particles involved and $s$. We take advantage of the frame-independence of this calculation and compute cross-sections in the center of mass frame. Taking $s=4 m_{\chi}^{2} \gamma^{2}$, where $\gamma$ is the

\footnotetext{
${ }^{8}$ For a more detailed description of thermal freeze-out physics see for example section 3 of ref. [49].
} 
familiar Lorentz factor $\gamma=1 / \sqrt{1-\beta^{2}}$, with $\beta$ being the CoM DM velocity, with $T=T_{f}$, we can simplify the above expression to the form

$$
\left\langle\sigma v_{M ø l}\right\rangle=\frac{8 x_{f}}{K_{2}^{2}\left(x_{f}\right)} \int_{1}^{\infty} \sigma \gamma^{2}\left(\gamma^{2}-1\right) K_{1}\left(2 x_{f} \gamma\right) d \gamma .
$$

All that remains to be determined are the individual annihilation cross sections $\sigma$.

These annihilation cross sections are calculated using the KK graviton Feynman rules found in refs. [37-41, 54], employing modifications to account for the factors of $\delta_{n}$ defined in eq. (2.7) which appear in front of the 5D Yang-Mills kinetic terms. Similarly, for the various chiral fermions, we modify their couplings by factors of $c_{L, R}$ to account for their localization (brane vs. bulk) that can differ for SM doublet and singlet fields of the same flavor and which are accompanied by the projection operators $P_{R, L}=\frac{1}{2}\left(1 \pm \gamma_{5}\right)$. In particular, we write the Feynman rule for the three point interaction between the $n^{\text {th }}$ KK graviton and two on-shell vector bosons as

$$
\frac{-i}{\sqrt{2} \Lambda_{\pi}} \delta^{a b} \lambda_{n}\left[m_{V}^{2} C_{\mu \nu, \rho \sigma}+\delta_{n}\left(k_{1} \cdot k_{2} C_{\mu \nu, \rho \sigma}+D_{\mu \nu, \rho \sigma}\left(k_{1}, k_{2}\right)\right)\right],
$$

where $C_{\mu \nu, \rho \sigma}$ and $D_{\mu \nu, \rho \sigma}\left(k_{1}, k_{2}\right)$ are defined in the appendix of ref. [54], $k_{1}$ and $k_{2}$ are the 4-momenta of the respective vector bosons, $m_{V}$ is the mass of the vector boson, $\lambda_{n}$ is the coupling strength to the graviton defined above in eq. (2.5), and $\delta^{a b}$ is the Kroenecker delta function of the gauge indices $a$ and $b$ of the vector bosons. Note that the factor of $\delta_{n}$ only modifies the terms in the Feynman rule arising from the Yang-Mills kinetic term, and it does not modify the term that is proportional to the boson mass, which is generated via spontaneous symmetry breaking on the IR-brane.

Next we consider the coupling of the KK graviton states to the chiral fermions. For the right-handed singlet $f_{R}$, we write the Feynman rule for the kinetic part of the on-shell three point interaction as

$$
\frac{-i}{2 \sqrt{2} \Lambda_{\pi}} \delta^{a b} c_{R, n} \lambda_{n} P_{R}\left[\gamma_{\mu}\left(k_{1 \nu}+k_{2 \nu}\right)+\gamma_{\nu}\left(k_{1 \mu}+k_{2 \mu}\right)\right],
$$

where $k_{1}\left(-k_{2}\right)$ is the 4 -momentum of the incoming (anti)fermion, $\delta^{a b}$ is the Kroenecker delta function for the fermion flavor indices, and $c_{R, n}$ is the modification to the coupling strength $\lambda_{n}$ arising from the possible localization of the right-handed fermion in the 5D bulk. In the case of a fermion residing on the TeV brane, $c_{R, n}=1$. The Feynman rule for the left-handed fermions is simply obtained by replacing $P_{R}$ and $c_{R, n}$ with $P_{L}$ and $c_{L, n}$. It is important to remember that the right- and left-handed chiral fermions may be located at different points in the $5 \mathrm{D}$ bulk, so that in general $c_{R, n} \neq c_{L, n}$. In addition to these kinetic pieces, there is also a coupling term on the IR-brane which corresponds to the fermion mass generated by spontaneous symmetry breaking via the Higgs vev and is simply proportional to $m_{f} \lambda_{n} \eta_{\mu \nu}$, linking the two chiral fermion fields.

Finally we consider the three point interaction between two incoming scalars and the $n^{\text {th }}$ KK graviton. We write the Feynman rule as

$$
\frac{-i}{\sqrt{2} \Lambda_{\pi}} \delta^{a b} \lambda_{n}\left(m_{s}^{2} \eta_{\mu \nu}-C_{\mu \nu, \rho \sigma} k_{1}^{\rho} k_{2}^{\sigma}\right)
$$


where $m_{s}$ is the mass of the scalar, $k_{1}$ and $k_{2}$ are the 4-momenta of the incoming scalars, and $\lambda_{n}, \eta_{\mu \nu}$ and $C_{\mu \nu, \rho \sigma}$ are previously defined. Note that since we only consider scalars being confined to the TeV brane, the Feynman rule in ref. [54] is only modified by a factor of $\lambda_{n}$.

Since all KK graviton states can mediate a given annihilation process, it is important to consider the effects of interference between the KK modes. The interference effects between the $i^{\text {th }}$ and $j^{\text {th }} \mathrm{KK}$ gravitons appear in the cross sections via the factor

$$
P_{i j}=\frac{\left(s-m_{G_{i}}^{2}\right)\left(s-m_{G_{j}}^{2}\right)+m_{G_{i}} \Gamma_{i} m_{G_{j}} \Gamma_{j}}{\left[\left(s-m_{G_{i}}^{2}\right)^{2}+m_{G_{i}}^{2} \Gamma_{i}^{2}\right]\left[\left(s-m_{G_{j}}^{2}\right)^{2}+m_{G_{j}}^{2} \Gamma_{j}^{2}\right]},
$$

where $m_{G_{i}}$ and $\Gamma_{i}$ are the mass and width of the $i^{t h}$ KK graviton. The cross sections contain the sum of these interference factors, $\sum_{i, j} P_{i j}$. When the KK gravitons have different coupling strengths, factors of $\lambda_{i}^{2} \lambda_{j}^{2}$ (along with factors of $c_{R / L, i} c_{R / L, j}$ for the case of chiral fermions or $\delta_{i} \delta_{j}$ for vector bosons) appear as well. The impact of the interference factors can be seen in figure 3 which shows the annihilation into two photons. In the case of the Brane model we have $\lambda_{i}=1$ for all $i$, and the interference minima are roughly evenly spaced between KK graviton resonances. When the KK gravitons couple to matter with different strengths, the interference minimum moves closer to the KK graviton resonance which couples more weakly to matter. In the case of the GW model we have $\lambda_{1}>\lambda_{2}>\lambda_{3}$ etc., so the interference minima are located near the heavier KK graviton resonance peaks.

The annihilation rates into the various SM final states contain velocity suppression factors resulting from the choice of spin of the DM particle. As an example, consider the cross sections for scalar, fermionic, and vector DM annihilating into a pair of Higgs bosons localized on the $\mathrm{TeV}$ brane (as given in eqs. (B.2), (B.6), and (B.12) of the appendix, respectively). For the case of scalar DM, $\sigma v$ is proportional to a factor of $\beta_{S}^{4}$, which corresponds to $\sim v^{4}$ in the center of mass frame. This represents so-called "d-wave" suppression of the annihilation since for spinless initial state DM we need to be in the $L=2$ angular momentum state to match the $J=2$ spin of the intermediate graviton KK state. Similarly, $\sigma v$ for fermionic DM annihilation is proportional to $\beta_{F}^{2}$, corresponding to $\sim v^{2}$ in the CoM, and is thus "p-wave" suppressed since the fermionic DM must be in the $L=1$ state to match with the KK graviton spin. In the case of vector DM, however, $\sigma v$ is not suppressed by any factors of $\beta_{V}$, as in this case annihilation can take place in the $L=0$ state or "s-wave" annihilation. It is important to note that these velocity-dependent suppressions are completely generic - all amplitudes for spin- $0(1 / 2,1)$ particles coupling to the graviton will experience an overall scaling of $\beta_{S}^{2}\left(\beta_{F}^{1}, \beta_{V}^{0}\right)$. Similar velocity suppressions are also present for the various SM final states, corresponding to their associated spins. E.g., in the case of annihilation to Higgs boson pairs, the cross section is always suppressed by a factor of $\beta_{h}^{5}$ (which includes the phase space correction) independent of the initial DM spin. Furthermore, in the simplest case where $S S \rightarrow h h$, so that both initial and final states must be in the d-wave, we not only see the appropriate velocity suppressions but also the factor of $\left(1-3 z^{2}\right)^{2}$ (where $z=\cos \theta$ being the CoM scattering angle). This corresponds to the square of the second order Legendre polynomial as expected as the $L=2$ orbital angular momentum wavefunction. This type of velocity scaling behavior is envisioned on general grounds based on the Breit-Wigner approximation. 


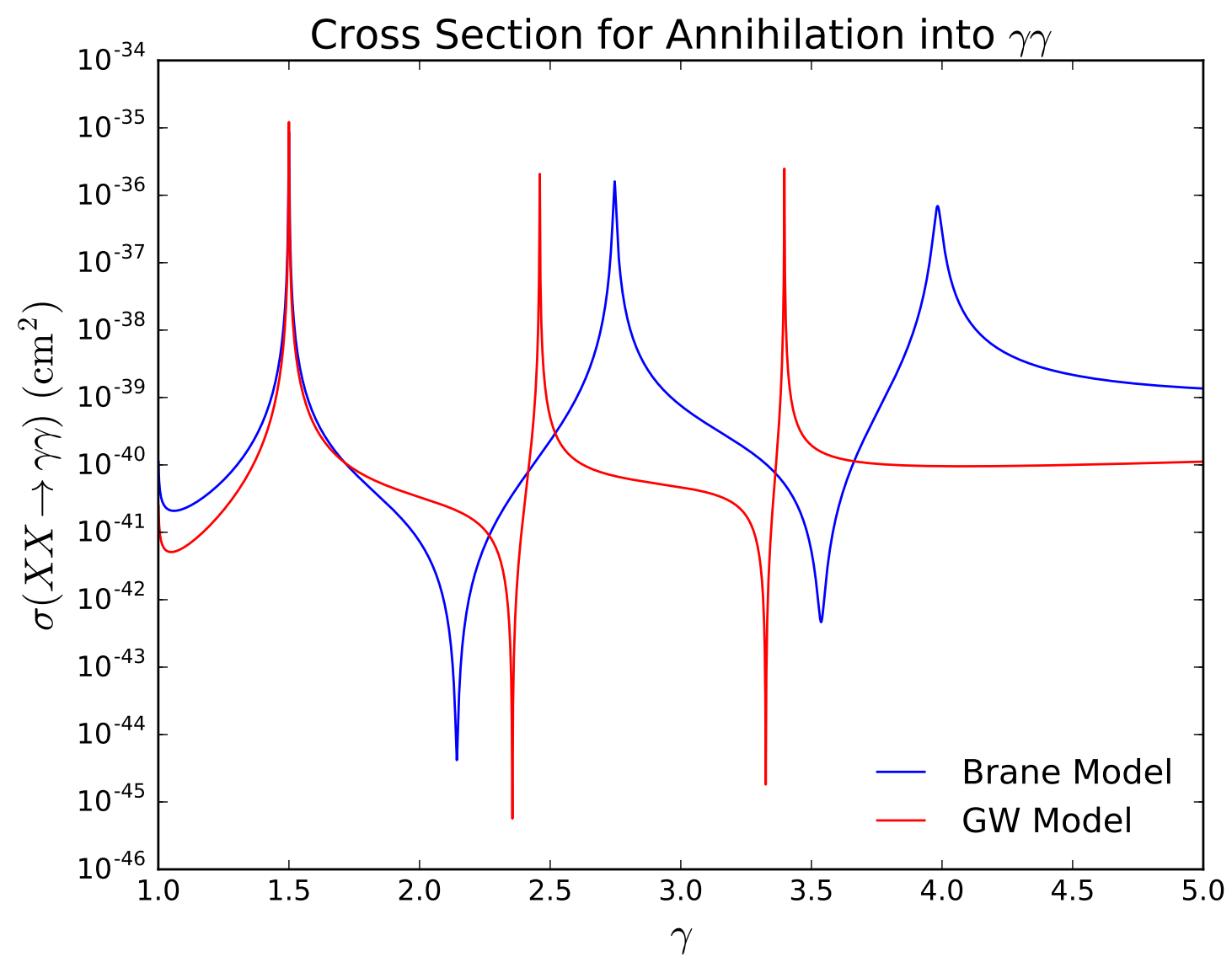

Figure 3. The cross section for spin-1 dark matter annihilating through $s$-channel exchange of the first $3 \mathrm{KK}$ gravitons as a function of the Lorentz factor $\gamma=\sqrt{s} / 2 m_{X}$ for the GW (red) and Brane (blue) models, taking $m_{X}=1000 \mathrm{GeV}$ and $m_{G_{1}}=3000 \mathrm{GeV}$. Note in particular that the weaker couplings of the higher KK gravitons to matter in the GW model push the maximal interference conditions close to the $s$-channel resonance peaks.

Expressions for the cross sections for DM annihilation into the various particles of the SM are given in appendix B. Given each of these individual contributions we can now calculate the inclusive $\left\langle\sigma v_{M ø l}\right\rangle$ for all of the various benchmark models described in section 2. We calculate the integral in eq. (3.4) numerically, using the VEGAS algorithm for adaptive Monte Carlo integration [55]. The results for each benchmark model are shown in figures 4 and 5.

We first examine the benchmark models with all the SM particles living on the IRbrane. The velocity suppression factors for the scalar and fermionic DM cases discussed above prevent these possibilities from saturating the observed relic abundance and creates the hierarchy of $\left\langle\sigma v_{M \varnothing l}\right\rangle$ values seen in figure 4 . Note that as the mass of the lightest graviton KK increases, the cross sections near KK resonances increase due to the nonrenormalizable graviton coupling. Thus while we see that the scalar and fermion DM scenarios never reach the desired value of the relic density for any of our benchmarks, we 

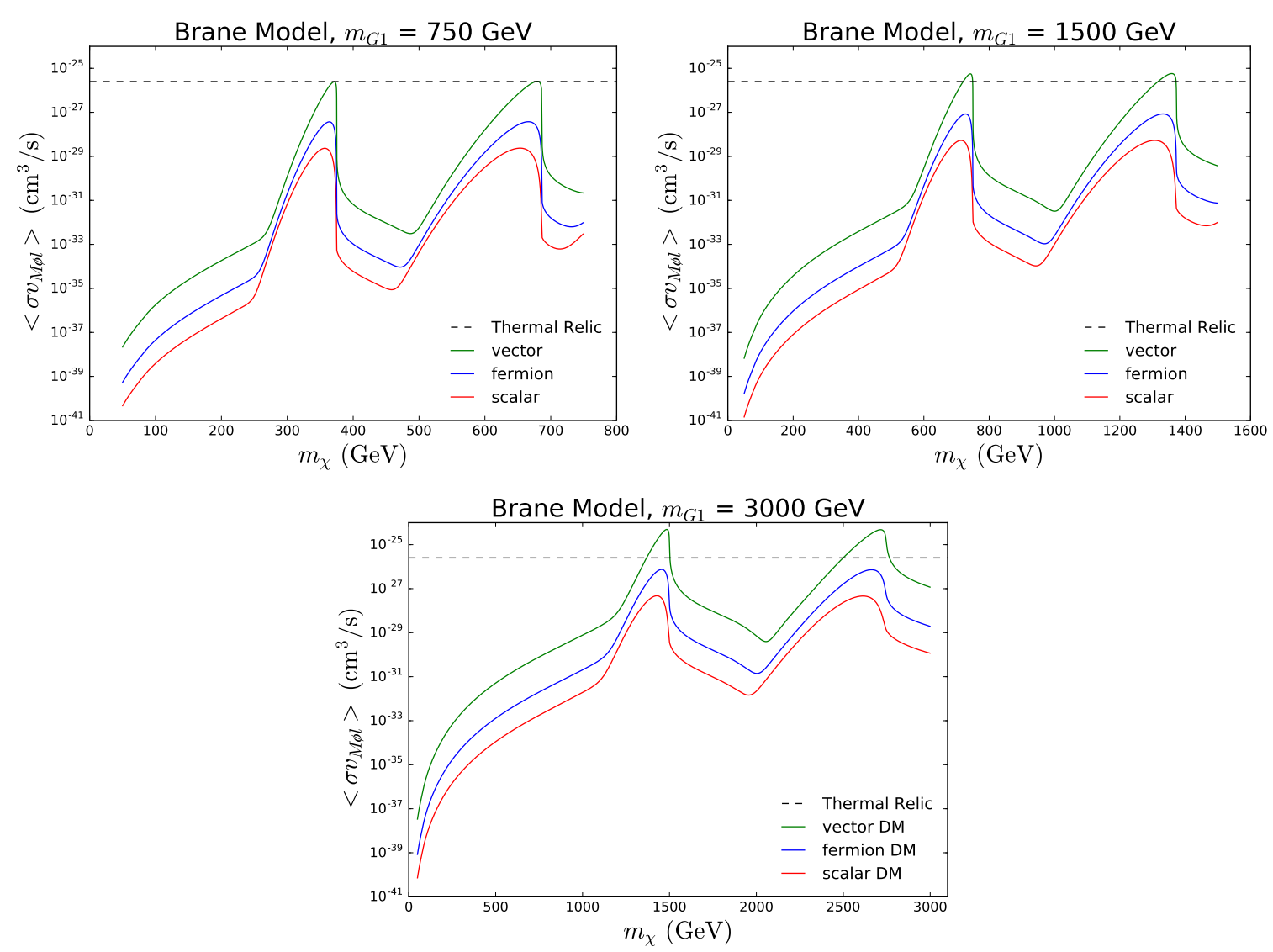

Figure 4. The thermally averaged cross section times Møller velocity for the benchmark models with all SM fields residing on the IR-brane as a function of the DM mass. The three benchmarks correspond to a lowest $\mathrm{KK}$ graviton mass of $750 \mathrm{GeV}$ (top left), $1500 \mathrm{GeV}$ (top right), and $3000 \mathrm{GeV}$ (bottom). The value of $\langle\sigma v\rangle$ associated with the observed relic abundance of DM is shown by the black dashed line.

note that this preferred value may be reached in the case of fermionic DM if the lightest KK graviton mass is increased to $4 \mathrm{TeV}$. However, for the specific IR-localized SM benchmarks that we have chosen the DM can only be spin-1, with mass near $m_{G_{n}} / 2$, in order to avoid over-closing the universe. We further observe that the second KK excitation peak cross section is quite close to that of the first KK state; we will return to this point below. It is also interesting to note that the benchmark scenarios that saturate the observed relic abundance only do so near the $s$-channel KK resonances where $m_{D M} \simeq m_{G_{n}} / 2$. The broadness of the peaks in $\left\langle\sigma v_{M ø l}\right\rangle$ arises from the Doppler broadening which occurs as part of the thermal averaging process - the widths of the KK gravitons themselves, as calculated in appendix A, are actually quite narrow. As an example, when $m_{G_{1}}=750 \mathrm{GeV}$, we find that $\Gamma_{G_{1}} \simeq 2 \mathrm{MeV}$, but the thermal averaging makes the resonance accessible to a significant amount of the DM thermal distribution for lower masses, thus broadening the first peaks in figure 4. The steep drop off in the annihilation rate at $m_{D M}=m_{G_{n}} / 2$ is a result of the Breit-Wigner peak in the cross section falling below the accessible center of mass energy, i.e., falling below $2 m_{D M}$. 
The MOR and GW benchmark models exhibit very similar behavior to the brane models discussed above, though the differing coupling strengths of the KK gravitons to matter create some important differences with respect to the thermal peaks. As in the IR-brane localized benchmark cases, we again see that only the possibility of spin-1 DM can reach the required thermal relic cross section although, increasing the lowest graviton KK mass may open up other possibilities. Here, in addition to the interference between KK modes being suppressed in the thermal averaging process as explained above, the relative peak heights within a single benchmark model are modified by the KK-number dependent, non-universal couplings. In particular, the peak heights of higher KK modes are reduced due to their smaller couplings to SM matter. This is most notable in the GW model shown in the right-hand panel of figure 5, where the second peak just rises to the observed thermal relic abundance cross section despite the first peak exceeding it by a substantial margin.

The interference effects between successive KK graviton states are also important to understand the shape of the various annihilation curves. In particular, the interference condition between the first two KK graviton modes is clearly visible in the Brane models as a strong dip in $\left\langle\sigma v_{M ø l}\right\rangle$ near $m_{D M} \sim 0.75 m_{G_{2}}$. This effect is most visible in the case of $m_{G_{1}}=3000 \mathrm{GeV}$ in figure 4 bottom. This feature is much less prominent in the MOR and GW models, shown in figure 5, due to the non-universal couplings. The Boltzmann factors in the thermal average suppress contributions of the cross section at high $s$, so only the low $s$ portion of the cross section contributes to the integral for a given DM mass. As a result, when there is large spacing (in $s$ ) between the interference minima and resonance maxima, it is possible to pick up a single minimum or maximum, which is what occurs in the Brane model with $m_{G_{1}}=3000 \mathrm{GeV}$ in figure 4 bottom. When the maxima and minima are pushed near one another due to KK gravitons coupling to matter with different strengths, the thermal average smears out the interference minimum with the resonance. This socalled Doppler broadening is responsible for the absence of dips in $\left\langle\sigma v_{M ø l}\right\rangle$ in the MOR and GW models in figure 5.

As already noted, all of these models are capable of saturating the observed relic abundance of dark matter in the case of spin-1 DM, as evidenced by the intersection of the green curves with the line corresponding to the thermal relic abundance cross section. We emphasize, however, that this only occurs for DM masses in the neighborhood of the $s$ channel resonances of the KK gravitons. The velocity suppression of the p-wave and d-wave annihilations (corresponding to fermionic and scalar DM, respectively) prevents them from achieving a sufficiently large value of $\left\langle\sigma v_{M \varnothing l}\right\rangle$ in all of our benchmark models, thus leading to a predicted overabundance of DM in these scenarios. Again we emphasize, however, that for larger values of the lightest KK graviton mass $m_{G_{1}}$ beyond our benchmark values, the fermion DM possibility (at least) can become a viable scenario for thermal DM.

\subsection{Dark matter indirect detection}

The properties of DM, as well as those of the KK gravitons in the models considered here, can in principle be constrained by searches for the various signatures that result from the DM annihilation process. Since DM annihilation proceeding through KK graviton exchange essentially leads to most, if not all, of the kinematically accessible SM particles 

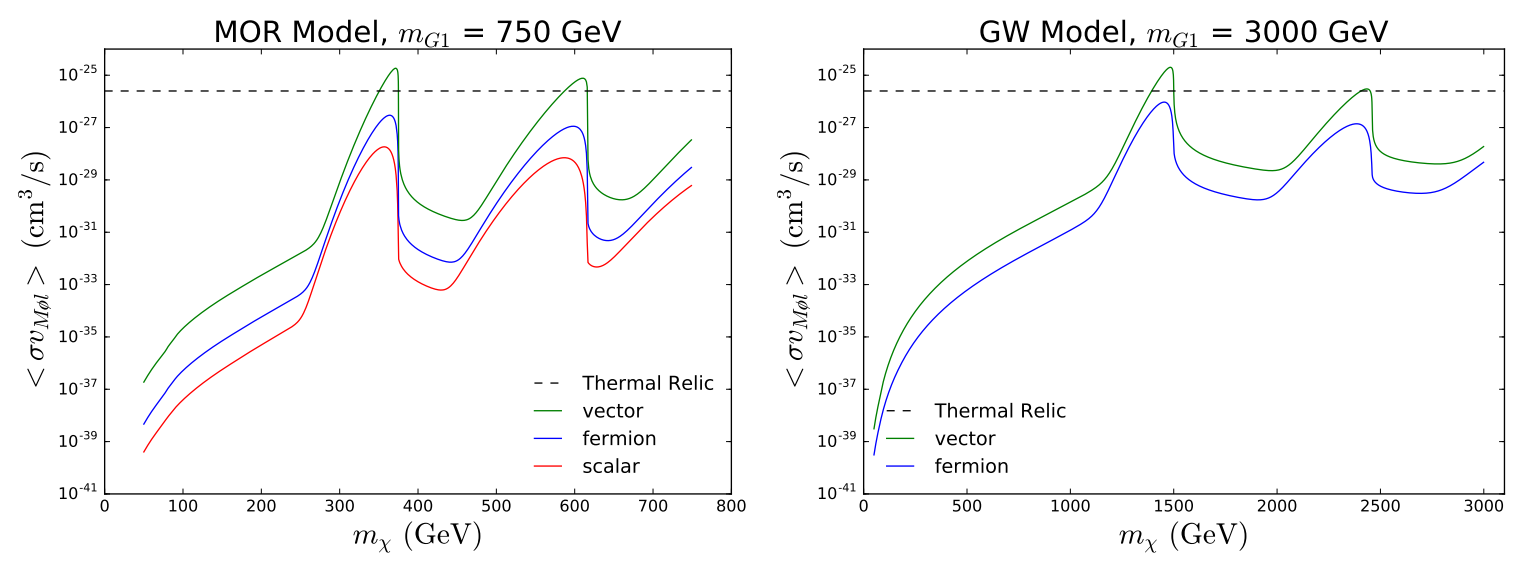

Figure 5. The thermally averaged cross section times Møller velocity for the MOR (left) and GW (right) benchmark models. The value of $\langle\sigma v\rangle$ associated with the observed thermal relic abundance of DM value is shown by the black dashed line.

with respectable branching fractions (BFs), there are numerous final states that can be employed in this endeavor. Here we will limit ourselves to the $\gamma \gamma, e^{+} e^{-}, \bar{p} p$ as well as the photon continuum (which follows from, e.g., the fragmentation process) final states as these potential signal channels cover most of the important graviton decay modes, and correspond to the experimental signatures. Interestingly, for the set of benchmark cases where all of the SM fields are confined to the IR-brane (and similarly for the GW scenario as well), apart from possible contributions that arise from decays to lighter gravitons that can occur for the higher KK excitations, the KK gravitons have the same BFs to the relevant SM fields, allowing for small phase space corrections. Thus, below the $G^{3} \rightarrow 2 G^{1}$ threshold and above top pair threshold, the ratio of, e.g., the DM annihilation branching rate into the diphoton final state is roughly a constant and is independent of the spin of the DM. This ratio can be identified with the value of $B\left(G^{1} \rightarrow 2 \gamma\right) \simeq 0.042$ (in the case of IR-brane localization), as can be seen from a consideration of the Breit-Wigner approximation for the various $s$-channel annihilation cross sections employing running decay widths.

In order to access the impact of present-day indirect detection searches on our model parameter space, we must re-examine the thermal relic calculation of $\left\langle\sigma v_{M ø l}\right\rangle$ performed above. The reason for this is clear: instead of being semi-relativistic at freeze-out, the DM of interest to us at present times is gravitationally bound in galaxies and is quite non-relativistic, i.e., $v \sim 10^{-3}$. The present time $\mathrm{DM}$ velocity distribution roughly takes the form of a truncated Maxwellian

$$
f(v) \sim v^{2} e^{-3 v^{2} / 2 v_{0}^{2}} \theta\left(v_{\max }-v\right),
$$

where $v_{0}$ and $v_{\max }$ are both typically of $O\left(10^{-3}\right)$, and $\theta(x)$ is the Heaviside theta function. (Here, for numerical purposes in our calculations below we take $v_{0}\left(v_{\max }\right)=150(550)$ $\mathrm{km} / \mathrm{sec}$. [56], although this specific choice of values turns out not to be very important as discussed below.) However, in practical applications, one finds that since the typical value of the velocity is sufficiently small, the approximation $v \rightarrow 0$ is an excellent one in 
performing the necessary cross section calculations below for the case of spin- 1 DM. ${ }^{9}$ Due to the large $\sim v^{2}\left(v^{4}\right)$ suppression factors experienced in the spin-1/2(0) DM scenarios, the annihilation cross sections at present times for these cases are highly suppressed by many orders of magnitude and do not reach the required thermal cross sections even at freeze-out. The resulting annihilation rates in these two cases for all of the various signal final states are found to be very far below those probed by the current indirect search experiments (as we will see below) and so are not constrained by them, or by any foreseeable improvements in these searches in the near future. Thus we need not consider these two spin cases any further here. Let us now turn to a discussion of the indirect detection final states relevant for experiment, and focus our attention on the spin-1 DM case. As we will discover, independent of the search channel, these constraints are extremely weak at best.

The $\gamma \gamma$ 'photon-line' search mode is potentially more important for the KK gravitonmediated DM annihilation process than in the case of, e.g., neutralinos in Supersymmetry where such a final state is only achievable via 1-loop graphs and correspondingly has a highly suppressed diphoton branching fraction. As noted above, by contrast, the diphoton branching fraction for KK gravitons lie in the range of $\sim 4-8 \%$, depending upon how the various SM fields are localized. The existing limits on this mode arise from both the FermiLAT [5] and HESS [6, 7] Galactic Center (GC) data sets, with the actual constraints being strongly dependent upon whether a cored or cusped DM profile is assumed to be applicable at the GC. Future measurements by CTA [8] are expected to significantly improve the existing constraints over a reasonable DM mass range. In figures 6 and 7 we compare these observational constraints for this final state with the present day $(z=0) \mathrm{DM}$ annihilation rates, and, for contrast, the rate at thermal freeze-out for our five benchmark models, assuming both cusped and cored DM profiles. These sets of distributions have several features in common: as already noted above, the Doppler broadening in the region of the graviton resonance peaks produce a rather wide maximum in the annihilation cross section during freeze-out. Today, DM is so slowly moving that $v \simeq 0$ is a good approximation in which case the width of the resonance peak is now set by that of the graviton KK resonances themselves, which are quite narrow for all 5 of our benchmarks. In the case of DM with a cusped profile, it is clear that these constraints can potentially be important in the very narrow mass range around the resonances for all 5 benchmark models, however, most of the parameter space allowing for the observed relic density is not impacted by these constraints. On the other hand, employing the cored DM profile yields no constraints from these observations. The reason for this is the predicted photon flux from the GC is proportional to the square of the DM density integrated over the region of interest on the sky, and so is much reduced in the case of a cored profile. However, for either choice of DM profile, the impact of these searches on our parameter space is at most quite minimal and only very close to a KK resonance. We note that improved sensitivity is expected in the future from searches at CTA.

A second constraint can arise from searches for an excess in the gamma ray continuum from dwarf galaxies; here we will make use of the experimental results as presented in

\footnotetext{
${ }^{9}$ We have explicitly checked that employing either $v=0$ or the distribution above with finite DM velocities, produces the same result in this case at the $\sim 1 \%$ level.
} 

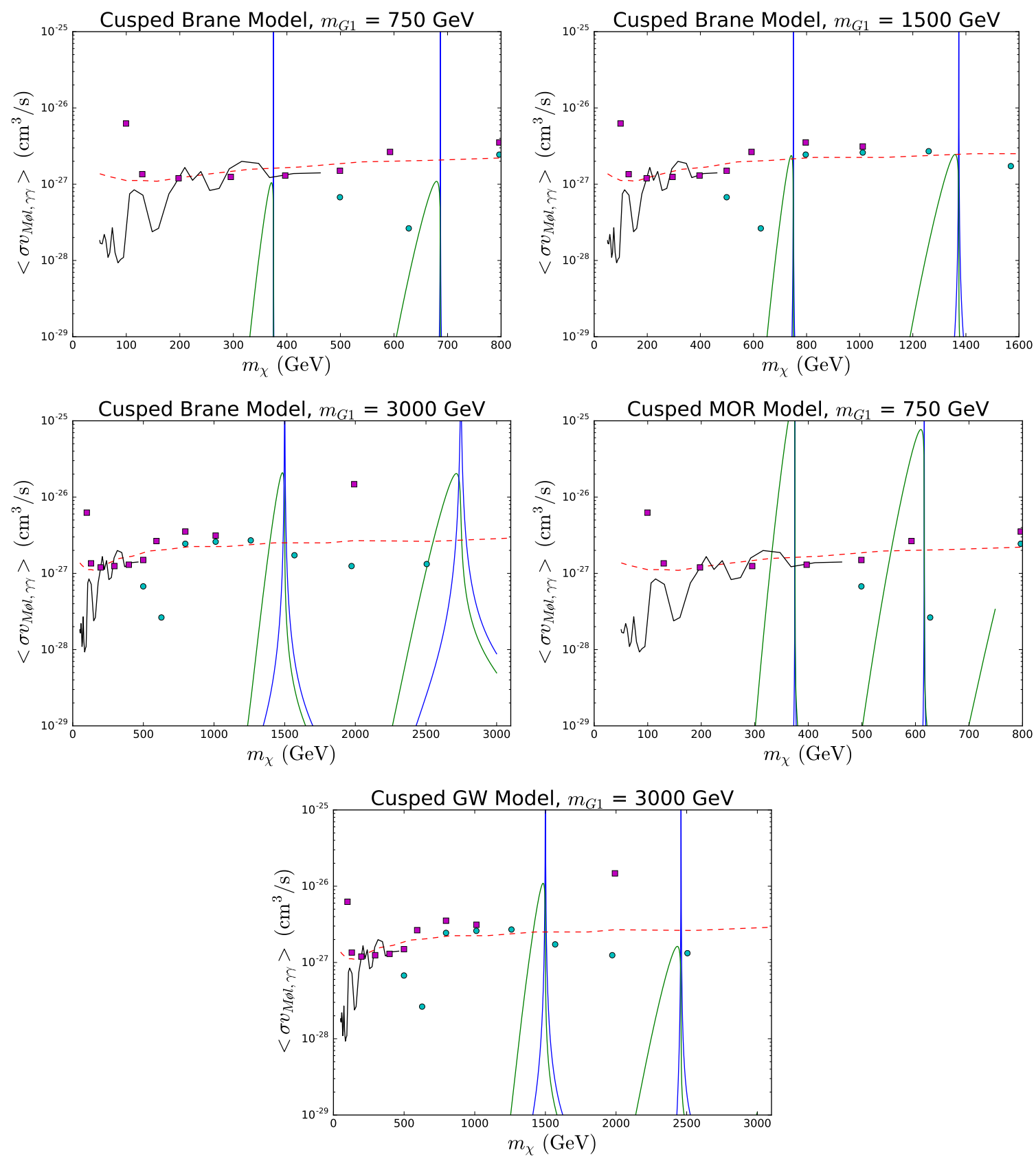

Figure 6. Assuming a cusped profile, the thermally averaged dark matter annihilation cross section into two photons at $z=0$ (at thermal freeze-out) for spin-1 dark matter as a function of the DM mass is given by the blue (green) curve for the 5 benchmark models as labeled. The photon line search limits from FERMI (solid black), HESS I (cyan circles), and HESS II (magenta squares) are also shown, as well as the projected sensitivity of the Cerenkov Telescope Array (red-dashed line). 

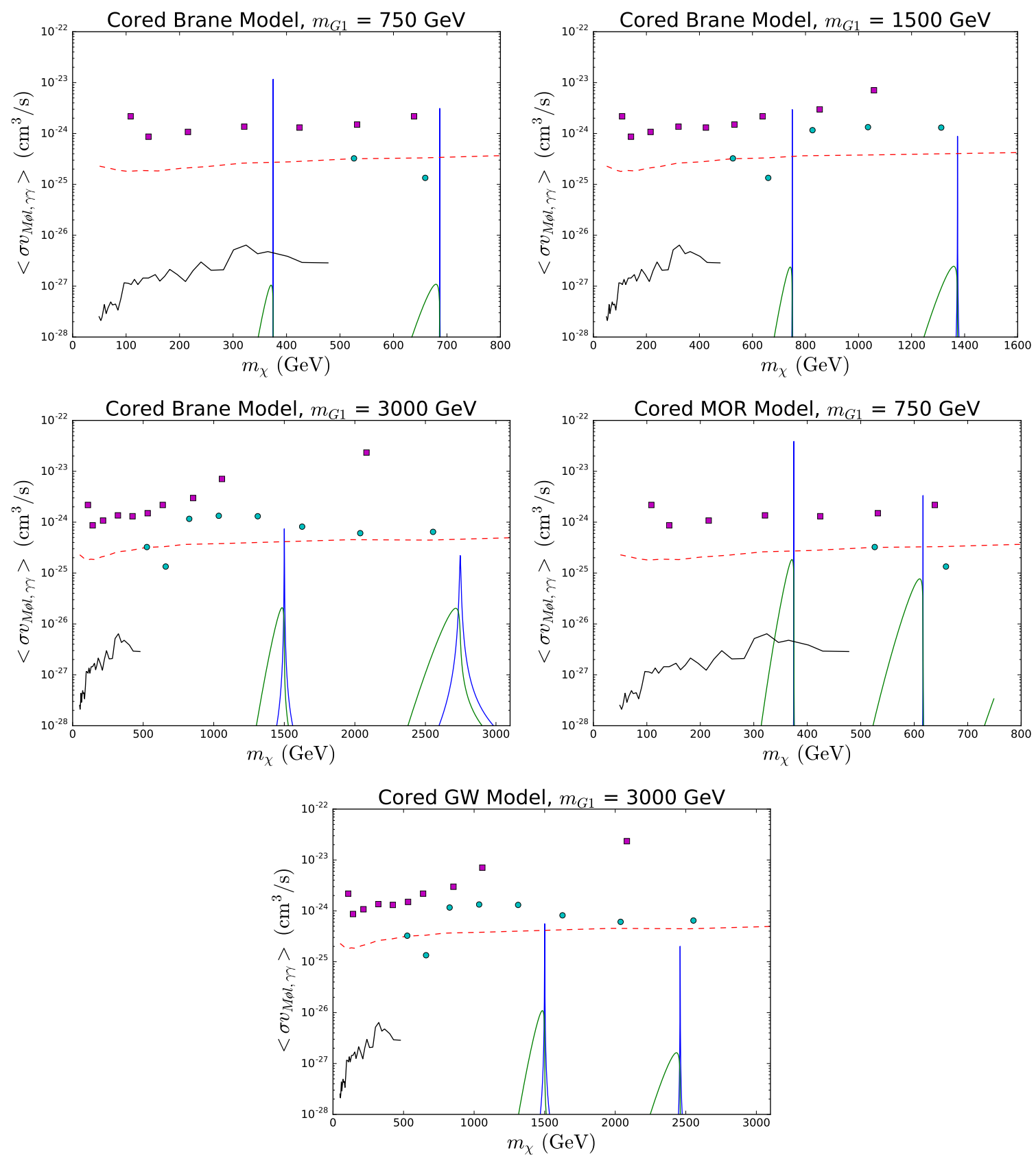

Figure 7. Assuming a cored profile, the thermally averaged dark matter annihilation cross section into two photons at $z=0$ (at thermal freeze-out) for spin-1 dark matter as a function of the DM mass is given by the blue (green) curve for the 5 benchmark models as labeled. The photon line search limits from FERMI (solid black), HESS I (cyan circles), and HESS II (magenta squares) are also shown, as well as the projected sensitivity of the Cerenkov Telescope Array (red-dashed line). 
refs. [9-12] employing dwarf stacking. The quoted limits assume that the DM annihilates predominantly into a particular SM final state, e.g., $\bar{b} b, W^{+} W^{-}$or $\tau^{+} \tau^{-}$, which is not the case for the present scenario due to the essentially universal nature of the graviton KK couplings. ${ }^{10}$ As can be seen from these references, the $\bar{b} b$ final state provides the strongest constraint, however, even if it were to be the dominant annihilation mode there would be essentially no impact on any of our scenarios. The strongest bound we find is for the case of a $3 \mathrm{TeV} G_{1} \mathrm{KK}$ excitation, where in the resonance region the cross section is constrained to be less than $\simeq 2.8 \times 10^{-25} \mathrm{~cm}^{3} \mathrm{~s}^{-1}$. However, due to the very narrow nature of the DM annihilation resonance peak for $z=0$ essentially none of the parameter space is excluded by this measurement. Of course in the more realistic situation where the limit from photon continuum is a weighted combination of the various SM final states, this constraint is further weakened by at least a factor of a few in comparison to the full $\bar{b} b$ channel. Thus we conclude that the photon continuum measurements are unlikely to provide constraints on gravity mediated DM annihilation.

A third possible channel which may place constraints on this scenario arises from the bound on the $\bar{p}$ flux; these particles are produced as a result of the decay and fragmentation of the SM annihilation products in a manner similar to that of the continuum gamma rays. There are two recent theoretical analyses of this bound $[57,58]$ employing the latest measurements from AMS-02 [59] which we make use of in our analysis. ${ }^{11}$ As in the case of continuum gammas, the theoretical estimate for the $\bar{p}$ flux is somewhat dependent upon which SM final state dominates the DM annihilation process. However, one might expect that most non-leptonic final states will produce rather similar bounds; in particular, ref. [57] shows explicitly that the assumption of dominance of either the $\bar{b} b$ or $W^{+} W^{-}$channels will lead to similar constraints on the $\bar{p}$ flux. As these authors note in particular, all of the non-leptonic SM final states yield very similar $\bar{p}$ spectra and so the constraints they yield are robust unless leptonic final states play a significant role in the DM annihilation process. Away from the DM signal region, the bounds that these authors obtain on the DM velocity-weighted annihilation cross section is a relatively smooth function of the DM mass. Allowing for the systematic uncertainties presented in figure 7 of ref. [57], and treating the $\bar{p}$ yield for all of the KK graviton annihilation products identically, we find the results for the $5 \mathrm{RS}$ benchmark models as summarized in figure 8 . To obtain these results we included annihilation to all final states except for photons and leptons with the approximation that the remaining modes produce similar $\bar{p}$ spectra. As in the cases above, we display the annihilation cross sections for $z=0$, as well as those during freeze-out for comparison. Here we see that for all of the benchmark models, as might be expected, the only constraint on the parameter space lies in the narrow graviton KK resonance regions. In some cases, e.g., for the GW benchmark, even this very weak constraint is seen to apply only to the region near the first KK excitation resonance. Thus, as was the case for the

\footnotetext{
${ }^{10}$ As noted above, the continuum photons, for the non-leptonic annihilation modes, are the result of the hadronization of the decay products of the various SM final states.

${ }^{11}$ Perhaps most interestingly, both of these theoretical analyses of the AMS-02 data strongly favor an excess consistent with the annihilation of $\sim 50-80 \mathrm{GeV}$ DM into $\bar{b} b$ or $W^{+} W^{-}$with essentially the thermal cross section reminiscent of the FERMI GC excess.
} 

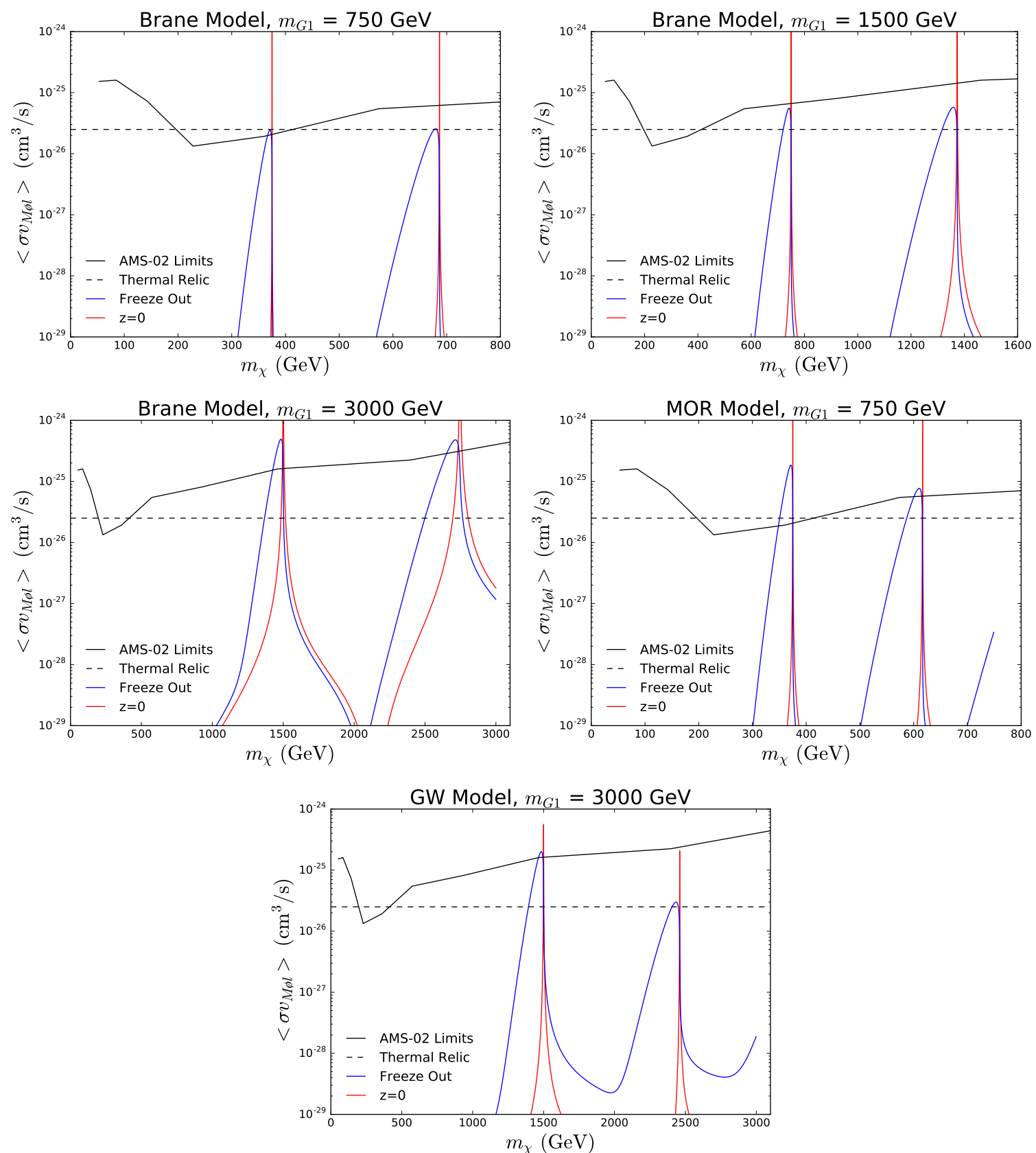

Figure 8. The thermally averaged annihilation cross sections for spin-1 dark matter annihilating into $p \bar{p}$ at $\mathrm{z}=0$ (red) and at freeze-out (blue) in the 5 benchmark models as a function of the DM mass. The AMS-02 antiproton constraints correspond to the black curve, while the value of $\langle\sigma v\rangle$ that achieves the observed relic abundance is shown as the dashed black curve as a guide for allowed DM masses.

other final states discussed above, these searches provide little if any constraint on RS gravity mediated DM annihilation for the parameter regions discussed here.

Finally, we consider the possible bounds arising from a potential excess in the flux of positrons due to DM annihilation, following ref. [60], which makes use of the data from 
AMS-02 [61-63]. Here, the positrons can either be directly produced as DM annihilation products or can be the result of decays and fragmentation of the other SM final states. The various constraints for the different annihilation products are summarized in ref. [60]. In all cases we see that these constraints remain sufficiently loose that none of our benchmark model points lie close to the corresponding bounds even if the channel with the largest rate is assumed to be applicable. Thus no further constraints on our RS gravity mediated benchmark models are obtained from this measurement.

\subsection{Dark matter spin-independent direct detection}

For completeness, we examine the cross section relevant for direct detection with KK graviton mediation. To begin this discussion we recall the tensor structure of the graviton exchange interaction between two conserved, stress-energy sources, $T_{\mu \nu}^{(1,2)}$; schematically we can write this amplitude as proportional to the quantity

$$
\mathcal{M} \sim T_{\mu \nu}^{1} P^{\mu \nu \alpha \beta} T_{\alpha \beta}^{2},
$$

where $P$ is the polarization tensor sum that appears in the numerator of the graviton propagator [54]. We can rewrite this amplitude in the form (recalling that the stressenergy tensor sources are conserved)

$$
\mathcal{M} \sim 2 T^{1 \mu \nu} T_{\mu \nu}^{2}-\frac{2}{3} T^{1} T^{2},
$$

where $T^{i}=\eta^{\mu \nu} T_{\mu \nu}^{i}$ is the trace of the energy-momentum tensor $T_{\mu \nu}^{i}$. It is convenient to recast this amplitude in terms of the trace and traceless components, $\tilde{T}_{\mu \nu}=T_{\mu \nu}-\frac{1}{4} \eta_{\mu \nu} T$, of the stress-energy tensor:

$$
\mathcal{M} \sim 2 \tilde{T}^{1 \mu \nu} \tilde{T}_{\mu \nu}^{2}-\frac{1}{6} T^{1} T^{2}
$$

This form is particularly useful when considering the direct detection (DD) of DM scattering off nuclei, since the nucleon matrix elements of both the trace and traceless parts of the stress-energy tensor are well-known. For simplicity let us consider first the case where all the SM fields lie on the TeV brane. Here, the stress-energy tensor sums over the set of the quark and gluon components of the nucleon, all of which couple to the graviton KK tower fields with the same strength. Summing over these components yields unambiguous nucleon matrix elements for both the trace and traceless parts of the stress-energy tensor, ${ }^{12}$ i.e., $<N\left|T_{N}\right| N>=2 m_{N}^{2}$ and $<N\left|\tilde{T}_{\mu \nu}^{N}\right| N>=2\left(k_{\mu} k_{\nu}-\frac{1}{4} \eta_{\mu \nu} m_{N}^{2}\right)$, where $m_{N}$ is the nucleon mass and $k_{\mu}$ is the corresponding nucleon 4-momentum. Here we have used the fact that the stress-energy tensor is conserved, i.e., $q_{\mu, \nu} T^{\mu \nu}=0$, where $q_{\mu}$ is the virtual 4-momentum carried by the exchanged KK graviton tower between the DM and the nucleus. In order to compute the scattering cross section we take the non-relativistic limit for the DM in the lab frame and neglect any small, velocity-dependent corrections. Denoting the KK tower sum as $m_{G R}^{-2} \equiv \sum_{i} m_{i}^{-2}$ where $m_{i}$ are the $i^{t h} \mathrm{KK}$ tower member graviton masses, we find

\footnotetext{
${ }^{12}$ See for example ref. [64].
} 
that the spin-independent (SI) DM-nucleon direct detection cross section for scalar, Dirac fermion or vector DM cases discussed above can all be written as

$$
\sigma_{N}=\frac{1}{9 \pi}\left[\frac{\mu}{m_{N}}\right]^{2}\left[\frac{m_{N}}{m_{G R} \Lambda_{\pi}}\right]^{4} m_{D M}^{2},
$$

where $\mu$ is the DM-nucleon reduced mass of order $m_{N}$ for DM masses of interest to us. Note that since we neglect the velocity-dependent corrections and average over the DM spin states, $\sigma_{N}$ is independent of the DM spin. Recalling that DM masses in the vicinity of $\simeq m_{i} / 2$ (for some value of $i$ ) are needed to saturate the relic density, we find that in all cases $\sigma_{N}$ lies very far below the current LUX/PandaX search limits [3, 4] for any interesting values of the parameters, e.g.

$$
\sigma_{N} \simeq 3.3 \cdot 10^{-54} \mathrm{~cm}^{2}\left[\frac{m_{D M}}{500 \mathrm{GeV}}\right]^{2}\left[\frac{30 \mathrm{TeV}^{2}}{m_{G R} \Lambda_{\pi}}\right]^{4} .
$$

Furthermore, these cross sections are sufficiently small that they are not likely to be probed by direct detection anytime in the near future with any of the planned experiments. Unfortunately, in the cases where the KK gravitons effectively decouple from the light quarks and couple only to the gluons in the nucleon, as in both the GW and MOR benchmarks, these results will be smaller by roughly an additional order of magnitude.

\section{Discussion and conclusions}

In this paper we have examined the possibility that WIMP-like dark matter interacts solely through gravitational interactions, with the observed relic density being achieved via annihilation into SM fields as mediated by Kaluza-Klein graviton excitations within the context of the Randall-Sundrum model. Five benchmark scenarios within this framework were considered in detail corresponding to various values for the mass of the first graviton KK excitation, differing SM matter localizations and distinct values of their brane localized kinetic terms. The DM candidate was assumed to be a SM singlet with the following three choices of spin being considered: a real scalar $(s=0)$, a vector-like neutral Dirac fermion $(s=1 / 2)$ or a real $\mathrm{U}(1)$ massive vector gauge field $(s=1)$. The annihilation cross sections and signals for both indirect and direct detection were studied in detail.

Independently of the nature of the SM final state, the velocity weighted annihilation cross sections for all scenarios were found to scale as $\sim v^{4(1-s)}$, where $v$ is the DM velocity in the DM center of mass frame. Since the DM is essentially non-relativistic at freeze-out implying small velocities, the annihilation of vector DM is then less velocity suppressed and naturally yields a substantially larger cross section than do either scalar or fermionic DM. This scaling with the DM velocity reflects the $\mathrm{d}(\mathrm{p}, \mathrm{s})$-wave behavior of the annihilation for the various spin possibilities given the spin-2 nature of the intermediate graviton KK excitations. For all benchmark models, vector DM is the only scenario that was found to be capable of reaching velocity-weighted annihilation cross sections in the range required to obtain the observed relic density for thermal DM. However, even in this case, the required 
annihilation rate is only obtained in the general neighborhood of one of the graviton KK resonances, i.e., for $m_{D M} \simeq 0.5 m_{G_{n}}$. We note, however, that for a somewhat more massive first graviton KK state, for example $\sim 4-5 \mathrm{TeV}$, a window of opportunity to generate the observed relic density opens for the case of thermal fermionic DM.

Direct detection searches for DM in the spin-independent channel (as is relevant for this scenario) were found to be essentially insensitive to this framework with both current and expected sensitivities being very far from the predicted direct detection cross section. The main reason for this small cross section is that the effective operator generated by the graviton KK resonances is of dimension- 8 since both the couplings of the SM and DM fields to these gravitons are non-renormalizable.

On the other hand, indirect searches for DM could potentially have an impact on small regions of the RS parameter space where larger annihilation rates can be obtained. This region occurs for spin-1 DM with masses extremely close, i.e., within a graviton KK width, of half the graviton KK mass $m_{D M}=m_{G_{n}} / 2$. Unlike in the case of thermal relic annihilation at freeze-out, where Doppler broadening widens the effective widths of the KK resonance peaks while decreasing their heights, present day velocity-weighted annihilation cross sections (which occur with essentially $v_{D M}=0$ ) lead to resonance peaks with widths that are determined only by the couplings of the KK graviton resonances themselves. Thus while a significant range of DM masses can generate the observed relic density, only very narrow DM mass windows are subject to present-day indirect searches. For these very narrow parameter regions searches for, e.g., gamma ray lines can be of potential importance since the diphoton decay branching fraction of our benchmark model points always exceeds $\sim 4 \%$. However, the anticipated fluxes of these photons are quite sensitive to the nature of the DM distribution at the galactic center, and for the case of a cored DM profile even these very weak constraints near the KK resonances can be further diminished. Even granting this uncertainty in the DM galactic center distribution, we can conclude that photon line searches do not significantly impact this scenario outside of extremely narrow DM mass regions. In a similar manner, we find that searches for DM induced excesses in either the gamma ray continuum or for the positron flux are found to yield no additional constraints. Other possible constraints arising from searches for a DM induced excess in the $\bar{p}$ flux can potentially be as, or more, important than in the case of photon line searches. However, using the results from two recent theoretical analyses of the AMS-02 data we found that, except in the regions very close to the graviton KK resonances, these constraints are again found to have essentially no impact on the RS parameter space.

The Randall-Sundrum framework provides a natural mechanism for thermal DM annihilation to achieve the observed relic density purely through gravitational interactions. DM detection is difficult in this scenario, and its best test remains the collider production of graviton KK resonances.

\section{Acknowledgments}

The work was supported by the Department of Energy, Contract DE-AC02-76SF00515. 


\section{A Cross sections and width for graviton-mediated annihilation: graviton width}

We begin by giving expressions for the KK graviton partial widths into scalars, fermions, and vectors (with the appropriate suppression factors $\delta$ for the transversely polarized fields which live in the bulk). Note the dark matter always lives on the $\mathrm{TeV}$ brane except for the GW benchmark point where it is localized similar to the SM $Z$ or $t_{R}$ depending upon its spin. The width into real scalars and fermions, respectively, are

$$
\begin{aligned}
\Gamma_{s s} & =\frac{\lambda^{2} m_{G}^{3}}{960 \pi \Lambda_{\pi}^{2}}\left(1-4 r_{s}\right)^{\frac{5}{2}}, \\
\Gamma_{f \bar{f}} & =N_{c} \frac{\lambda^{2} m_{G}^{3}}{160 \pi \Lambda_{\pi}^{2}}\left(1-4 r_{f}\right)^{\frac{3}{2}}\left[\frac{10}{3} r_{f}+\frac{\left(c_{L}^{f}\right)^{2}+\left(c_{R}^{f}\right)^{2}}{2}\left(1-\frac{2}{3} r_{f}\right)\right],
\end{aligned}
$$

where $r_{x}=m_{x}^{2} / m_{G}^{2}$ and $N_{c}$ is the usual color factor. $\lambda=1$ for models with all SM fields on the IR-brane and $\simeq 0.126$ for the MOR benchmark with bulk fields. The factors $c_{L, R}^{f}$ are described in the text above and account for the localizations of the left- or right-handed SM fermions in the bulk. For the massless gauge bosons, we find the partial width to be

$$
\Gamma_{g g, \gamma \gamma}=N_{c} \delta^{2} \frac{\lambda^{2} m_{G}^{3}}{80 \pi \Lambda_{\pi}^{2}}
$$

where $N_{c}=8$ for $g g$ and 1 for photons. $\delta=1$ for gauge bosons which live on the IR-brane, $1 / 2$ for gauge bosons which live in the bulk for the MOR benchmark, while in the GW scenario one has $\lambda \delta \simeq 1$. For the massive SM gauge bosons, we find the corresponding partial widths:

$$
\Gamma_{V V}=N \frac{\lambda^{2} m_{G}^{3} \sqrt{1-4 r_{V}}}{480 \pi \Lambda_{\pi}^{2}}\left(A_{V}+\delta B_{V}+\delta^{2} C_{V}\right)
$$

where

$$
\begin{aligned}
& A_{V}=1+12 r_{V}+56 r_{V}^{2}, \\
& B_{V}=80 r_{V}\left(1-r_{V}\right), \\
& C_{V}=12\left(1-3 r_{V}+6 r_{V}^{2}\right) .
\end{aligned}
$$

Here $\mathrm{N}=1$ for distinguishable particles $(V=W)$ and $1 / 2$ for identical particles $(V=Z)$ in the final state. Note that we keep the Higgs on the TeV brane in all models considered, so that the Goldstone modes of the massive gauge bosons will not pick up factors of $\delta$.

\section{B Dark matter annihilation cross sections}

\section{B.1 Scalar dark matter}

Here we consider the differential cross sections for annihilation of DM into the various SM final states. These processes are all mediated by the $s$-channel exchange of the massive KK graviton states. We begin with the case of scalar DM (denoted here by S). It is useful to 
define the quantity $P_{i j}$, which arises due to interference between the $i^{\text {th }}$ and $j^{\text {th }}$ KK mode exchanges,

$$
P_{i j}=\frac{\left(s-m_{i}^{2}\right)\left(s-m_{j}^{2}\right)+m_{i} \Gamma_{i} m_{j} \Gamma_{j}}{\left[\left(s-m_{i}^{2}\right)^{2}+m_{i}^{2} \Gamma_{i}^{2}\right]\left[\left(s-m_{j}^{2}\right)^{2}+m_{j}^{2} \Gamma_{j}^{2}\right]},
$$

where $m_{i}$ and $\Gamma_{i}$ are the mass and width of the $i^{t h}$ graviton KK mode, respectively. The first cross section we consider is the annihilation of scalar DM into a pair of Higgs bosons $(h)$,

$$
\frac{d \sigma_{S S \rightarrow h h}}{d z}=\frac{s^{3} \beta_{S}^{3} \beta_{h}^{5}\left(1-3 z^{2}\right)^{2}}{9216 \pi \Lambda_{\pi}^{4}} \sum_{i, j} \lambda_{i}^{2} \lambda_{j}^{2} P_{i j}
$$

where as usual $\beta_{x}=\sqrt{1-4 m_{x}^{2} / s}$ and $z=\cos (\theta)$, with $\theta$ being the scattering angle relative to the momentum axis of the incoming particles in the center of mass frame. In a similar fashion we find the differential cross section for $S S \rightarrow f \bar{f}$ to be

$$
\begin{aligned}
\frac{d \sigma_{S S \rightarrow f \bar{f}}}{d z}= & N_{c} \frac{s^{3} \beta_{S}^{3} \beta_{f}^{3}}{4608 \pi \Lambda_{\pi}^{4}} \times\left(\sum_{i, j} \lambda_{i}^{2} \lambda_{j}^{2} P_{i j}\right) \times \\
& \times\left[\left(1-\beta_{f}^{2}\right)\left(1+3 z^{2}\right)+\frac{c_{L, i}^{f} c_{L, j}^{f}+c_{R, i}^{f} c_{R, j}^{f}}{2}\left(1-\beta_{f}^{2}+3 z^{2}\left(1+5 \beta_{f}^{2}\right)-18 z^{4} \beta_{f}^{2}\right)\right],
\end{aligned}
$$

where the first term in brackets corresponds to the contribution from the Dirac mass term (arising from the Higgs vev on the IR-brane) and the second term corresponds to bulk left- and right-handed fermion fields. Here $N_{c}$ is the number of colors of the fermion, i.e., $=1$ (3) for leptons(quarks). When the SM Dirac fermion is restricted to the TeV brane, $c_{L}^{f}=c_{R}^{f}=1$. For the case of neutrinos on the TeV brane, we take $\beta_{f}=c_{L}^{f}=1$ and $c_{R}^{f}=0$. For photons and gluons in the final state, we find the differential cross-section to be

$$
\frac{d \sigma_{S S \rightarrow g g, \gamma \gamma}}{d z}=N \frac{s^{3} \beta_{S}^{3}\left(1-z^{2}\right)^{2}}{512 \pi \Lambda_{\pi}^{4}} \sum_{i, j} \lambda_{i}^{2} \lambda_{j}^{2} P_{i j} \delta_{i} \delta_{j}
$$

For $S S \rightarrow V V$ with massive SM gauge boson final states, we instead obtain

$$
\frac{d \sigma_{S S \rightarrow V V}}{d z}=N \frac{s^{3} \beta_{S}^{3} \beta_{V}}{4608 \pi \Lambda_{\pi}^{4}} \sum_{i, j} \lambda_{i}^{2} \lambda_{j}^{2} P_{i j}\left(A_{S V}+\frac{1}{2}\left(\delta_{i}+\delta_{j}\right) B_{S V}+\delta_{i} \delta_{j} C_{S V}\right)
$$

where

$$
\begin{aligned}
& A_{S V}=6+2 \beta_{V}^{2}\left(3 z^{2}-5\right)+\beta_{V}^{4}\left(5-12 z^{2}+9 z^{4}\right), \\
& B_{S V}=4\left(1-\beta_{V}^{2}\right)\left[3+\beta_{V}^{2}\left(2-3 z^{2}\right)\right], \\
& C_{S V}=6\left[1+\beta_{V}^{2}\left(1-3 z^{2}\right)+\beta_{V}^{4}\left(1-3 z^{2}+3 z^{4}\right)\right],
\end{aligned}
$$

and where as before $N=1$ for $W W$ and $1 / 2$ for $Z Z$. Note that $A_{S V}$ corresponds to the portion of the cross section proportional to the mass of the gauge boson generated via spontaneous symmetry breaking on the IR-brane, $C_{S V}$ corresponds to the cross section resulting from the pure 5-D Yang-Mills kinetic term, and $B_{S V}$ represents the interference between these two contributions. 


\section{B.2 Fermionic dark matter}

For the case of fermionic DM (denoted by $F$ ), we similarly compute the spin-averaged differential cross sections into the various SM final states. We begin with the result for $F \bar{F} \rightarrow h h$

$$
\frac{d \sigma_{F \bar{F} \rightarrow h h}}{d z}=\frac{s^{3} \beta_{F} \beta_{h}^{5}}{18432 \pi \Lambda_{\pi}^{4}}\left(1-\beta_{F}^{2}+3 z^{2}\left(1+2 \beta_{F}^{2}\right)-9 z^{4} \beta_{F}^{2}\right) \sum_{i, j} \lambda_{i}^{2} \lambda_{j}^{2} P_{i j} .
$$

Next we consider the process $F \bar{F} \rightarrow f \bar{f}$ for SM fermions $f$

$$
\frac{d \sigma_{F \bar{F} \rightarrow f \bar{f}}}{d z}=N_{c} \frac{s^{3} \beta_{F} \beta_{f}^{3}}{36864 \pi \Lambda_{\pi}^{4}} \sum_{i, j}\left(A_{f}+\frac{c_{L, i}^{f} c_{L, j}^{f}+c_{R, i}^{f} c_{R, j}^{f}}{2} B_{f}\right) \lambda_{i}^{2} \lambda_{j}^{2} P_{i j},
$$

where $A_{f}$ is generated from the Dirac mass term due to the Higgs on the IR-brane and $B_{f}$ arises from the left- and right-handed SM fields which may propagate in the bulk. $N_{c}$ is the same color factor as above. $A_{f}$ and $B_{f}$ are given by

$$
\begin{aligned}
& A_{f}=\left(1-\beta_{f}^{2}\right)\left(13-4 \beta_{F}^{2}+3 z^{2}\left(7-4 \beta_{F}^{2}\right)\right), \\
& B_{f}=13-4 \beta_{F}^{2}+\beta_{f}^{2}\left(5+4 \beta_{F}^{2}\right)+3 z^{2}\left(7-4 \beta_{F}^{2}-\beta_{f}^{2}\left(1+20 \beta_{F}^{2}\right)\right)+72 z^{4} \beta_{F}^{2} \beta_{f}^{2} .
\end{aligned}
$$

For the massless gauge boson final states, we find

$$
\frac{d \sigma_{F \bar{F} \rightarrow g g, \gamma \gamma}}{d z}=N \frac{s^{3} \beta_{F}}{1024 \pi \Lambda_{\pi}^{4}}\left(1-z^{2}\right)\left(2-\beta_{F}^{2}\left(1-z^{2}\right)\right) \sum_{i, j} \lambda_{i}^{2} \lambda_{j}^{2} P_{i j} \delta_{i} \delta_{j},
$$

where here $N=8$ for $g g$ and $N=1$ for $\gamma \gamma$. For the massive SM gauge bosons we instead find

$$
\frac{d \sigma_{F \bar{F} \rightarrow V V}}{d z}=N \frac{s^{3} \beta_{F} \beta_{V}}{18432 \pi \Lambda_{\pi}^{4}} \sum_{i, j} \lambda_{i}^{2} \lambda_{j}^{2} P_{i j}\left(A_{F V}+\frac{1}{2}\left(\delta_{i}+\delta_{j}\right) B_{F V}+\delta_{i} \delta_{j} C_{F V}\right),
$$

with

$$
\begin{aligned}
& A_{F V}= 6\left(5-2 \beta_{F}^{2}\right)-\beta_{V}^{2}\left(47-20 \beta_{F}^{2}+3 z^{2}\left(4 \beta_{F}^{2}-7\right)\right)+ \\
&+ \beta_{V}^{4}\left(19-10 \beta_{F}^{2}+3 z^{2}\left(8 \beta_{F}^{2}-5\right)-18 z^{4} \beta_{F}^{2}\right), \\
& B_{F V}=2\left(1-\beta_{V}^{2}\right)\left[30-12 \beta_{F}^{2}+\beta_{V}^{2}\left(17-8 \beta_{F}^{2}+3 z^{2}\left(4 \beta_{F}^{2}-7\right)\right)\right], \\
& C_{F V}=3\left\{10-4 \beta_{F}^{2}+\beta_{V}^{2}\left(3 z^{2}-1\right)\left(4 \beta_{F}^{2}-7\right)+\right. \\
&\left.+\beta_{V}^{4}\left(7-4 \beta_{F}^{2}-3 z^{2}\left(1-4 \beta_{F}^{2}\right)-12 z^{4} \beta_{F}^{2}\right)\right\},
\end{aligned}
$$

where $\mathrm{N}=1$ for $W W$ and $\mathrm{N}=1 / 2$ for $Z Z$ final states as before.

\section{B.3 Vector dark matter}

We now consider the spin-averaged differential cross sections for massive spin-1 DM which lives on the IR-brane. For the GW benchmark scenario with DM in the bulk, these expressions are easily adapted. We denote the DM here by $X$ (to avoid possible confusion with the massive SM gauge fields $V=W, Z$ ) and find the differential cross section for $X X \rightarrow h h$

$$
\frac{d \sigma_{X X \rightarrow h h}}{d z}=\frac{s^{3} \beta_{h}^{5}\left(24-8 \beta_{X}^{2}\left(1+3 z^{2}\right)+3 \beta_{X}^{4}\left(1-3 z^{2}\right)^{2}\right)}{82944 \pi \beta_{X} \Lambda_{\pi}^{4}} \sum_{i, j} \lambda_{i}^{2} \lambda_{j}^{2} P_{i j} .
$$


For final states containing SM fermions we find

$$
\frac{d \sigma_{X X \rightarrow f \bar{f}}}{d z}=N_{c} \frac{s^{3} \beta_{f}^{3}}{41472 \pi \beta_{X} \Lambda_{\pi}^{4}} \sum_{i, j}\left(C_{f}+\frac{c_{L, i}^{f} c_{L, j}^{f}+c_{R, i}^{f} c_{R, j}^{f}}{2} D_{f}\right) \lambda_{i}^{2} \lambda_{j}^{2} P_{i j},
$$

where $C_{f}$ is generated from the Dirac mass term due to the Higgs vev on the TeV brane and $D_{f}$ arises from left- and right-handed fields which may propagate in the bulk. $C_{f}$ and $D_{f}$ are given by

$$
\begin{aligned}
C_{f}= & \left(1-\beta_{f}^{2}\right)\left[60-2 \beta_{X}^{2}\left(13+21 z^{2}\right)+3 \beta_{X}^{4}\left(1+3 z^{2}\right)\right], \\
D_{f}= & 60-\beta_{X}^{2}\left(26+42 z^{2}\right)+3 \beta_{X}^{4}\left(1+3 z^{2}\right) \\
& +\beta_{f}^{2}\left[12-\beta_{X}^{2}\left(10-6 z^{2}\right)-\beta_{X}^{4}\left(3-45 z^{2}+54 z^{4}\right)\right] .
\end{aligned}
$$

For the massless gauge boson final states we find

$$
\frac{d \sigma_{X X \rightarrow g g, \gamma \gamma}}{d z}=N \frac{s^{3}\left(16-16 \beta_{X}^{2}\left(1-z^{2}\right)+3 \beta_{X}^{4}\left(1-z^{2}\right)^{2}\right)}{4608 \pi \beta_{X} \Lambda_{\pi}^{4}} \sum_{i, j} \lambda_{i}^{2} \lambda_{j}^{2} P_{i j} \delta_{i} \delta_{j},
$$

and for the massive SM gauge bosons we obtain

$$
\frac{d \sigma_{X X \rightarrow V V}}{d z}=N \frac{s^{3} \beta_{V}}{41472 \pi \beta_{X} \Lambda_{\pi}^{4}} \sum_{i, j} \lambda_{i}^{2} \lambda_{j}^{2} P_{i j}\left(A_{X V}+\frac{1}{2}\left(\delta_{i}+\delta_{j}\right) B_{X V}+\delta_{i} \delta_{j} C_{X V}\right),
$$

with

$$
\begin{aligned}
A_{X V}= & 6\left(30-20 \beta_{X}^{2}+3 \beta_{X}^{4}\right)-2 \beta_{V}^{2}\left[120-\beta_{X}^{2}\left(94-42 z^{2}\right)+3 \beta_{X}^{4}\left(5-3 z^{4}\right)\right]+ \\
+ & \beta_{V}^{4}\left[84-\beta_{X}^{2}\left(76-60 z^{2}\right)+3 \beta_{X}^{4}\left(5-12 z^{2}+9 z^{4}\right)\right], \\
B_{X V}= & 4\left(1-\beta_{V}^{2}\right)\left[90-60 \beta_{X}^{2}+9 \beta_{X}^{4}+\beta_{V}^{2}\left(30-\beta_{X}^{2}\left(34-42 z^{2}\right)+\beta_{X}^{4}\left(6-9 z^{2}\right)\right)\right], \\
C_{X V}= & 6\left\{30-20 \beta_{X}^{2}+3 \beta_{X}^{4}+\beta_{X}^{2} \beta_{V}^{2}\left(3 z^{2}-1\right)\left(14-3 \beta_{X}^{2}\right)+\right. \\
& \left.+\beta_{V}^{4}\left(18-2 \beta_{X}^{2}\left(7-3 z^{2}\right)+\beta_{X}^{4}\left(3-9 z^{2}+9 z^{4}\right)\right)\right\},
\end{aligned}
$$

with $\mathrm{N}=1$ for $W W$ and $\mathrm{N}=1 / 2$ for $Z Z$ as before.

Open Access. This article is distributed under the terms of the Creative Commons Attribution License (CC-BY 4.0), which permits any use, distribution and reproduction in any medium, provided the original author(s) and source are credited.

\section{References}

[1] G. Steigman and M.S. Turner, Cosmological constraints on the properties of weakly interacting massive particles, Nucl. Phys. B 253 (1985) 375 [INSPIRE].

[2] G. Bertone, D. Hooper and J. Silk, Particle dark matter: evidence, candidates and constraints, Phys. Rept. 405 (2005) 279 [hep-ph/0404175] [INSPIRE].

[3] LUX collaboration, D.S. Akerib et al., Results from a search for dark matter in the complete LUX exposure, Phys. Rev. Lett. 118 (2017) 021303 [arXiv: 1608.07648] [INSPIRE]. 
[4] PandaX-II collaboration, A. Tan et al., Dark matter results from first 98.7 days of data from the PandaX-II experiment, Phys. Rev. Lett. 117 (2016) 121303 [arXiv:1607.07400] [INSPIRE].

[5] Fermi-LAT collaboration, M. Ackermann et al., Updated search for spectral lines from galactic dark matter interactions with pass 8 data from the Fermi Large Area Telescope, Phys. Rev. D 91 (2015) 122002 [arXiv:1506.00013] [INSPIRE].

[6] H.E.S.S. collaboration, A. Abramowski et al., Search for photon-linelike signatures from dark matter annihilations with H.E.S.S., Phys. Rev. Lett. 110 (2013) 041301 [arXiv: 1301.1173] [INSPIRE].

[7] H.E.S.S. collaboration, H. Abdalla et al., H.E.S.S. limits on linelike dark matter signatures in the $100 \mathrm{GeV}$ to $2 \mathrm{TeV}$ energy range close to the galactic center, Phys. Rev. Lett. 117 (2016) 151302 [arXiv: 1609.08091] [INSPIRE].

[8] V. Lefranc, E. Moulin, P. Panci, F. Sala and J. Silk, Dark matter in $\gamma$ lines: galactic center vs dwarf galaxies, JCAP 09 (2016) 043 [arXiv: 1608.00786] [inSPIRE].

[9] Fermi-LAT collaboration, M. Ackermann et al., Searching for dark matter annihilation from milky way dwarf spheroidal galaxies with six years of Fermi Large Area Telescope data, Phys. Rev. Lett. 115 (2015) 231301 [arXiv:1503.02641] [INSPIRE].

[10] Figures and data files associated with the Fermi LAT paper "Searching for dark matter annihilation from milky way dwarf spheroidal galaxies with six years of Fermi-LAT data" webpage, http://www-glast.stanford.edu/pub_data/1048/.

[11] DES and Fermi-LAT collaborations, A. Drlica-Wagner et al., Search for gamma-ray emission from DES dwarf spheroidal galaxy candidates with Fermi-LAT data, Astrophys. J. 809 (2015) L4 [arXiv: 1503.02632] [INSPIRE].

[12] Fermi-LAT and MAGIC collaborations, M.L. Ahnen et al., Limits to dark matter annihilation cross-section from a combined analysis of MAGIC and Fermi-LAT observations of dwarf satellite galaxies, JCAP 02 (2016) 039 [arXiv:1601.06590] [INSPIRE].

[13] J. Alexander et al., Dark sectors 2016 workshop: community report, arXiv:1608.08632 [INSPIRE].

[14] ATLAS collaboration, Search for new high-mass resonances in the dilepton final state using proton-proton collisions at $\sqrt{s}=13$ TeV with the ATLAS detector, ATLAS-CONF-2016-045, CERN, Geneva Switzerland, (2016).

[15] ATLAS collaboration, Search for scalar diphoton resonances with $15.4 \mathrm{fb}^{-1}$ of data collected at $\sqrt{s}=13 \mathrm{TeV}$ in 2015 and 2016 with the ATLAS detector, ATLAS-CONF-2016-059, CERN, Geneva Switzerland, (2016).

[16] ATLAS collaboration, Search for new phenomena in dijet events with the ATLAS detector at $\sqrt{s}=13 \mathrm{TeV}$ with 2015 and 2016 data, ATLAS-CONF-2016-069, CERN, Geneva Switzerland, (2016).

[17] CMS collaboration, Search for resonant production of high mass photon pairs using $12.9 \mathrm{fb}^{-1}$ of proton-proton collisions at $\sqrt{s}=13 \mathrm{TeV}$ and combined interpretation of searches at 8 and 13 TeV, CMS-PAS-EXO-16-027, CERN, Geneva Switzerland, (2016).

[18] CMS collaboration, Search for a high-mass resonance decaying into a dilepton final state in $13 \mathrm{fb}^{-1}$ of pp collisions at $\sqrt{s}=13 \mathrm{TeV}$, CMS-PAS-EXO-16-031, CERN, Geneva Switzerland, (2016). 
[19] CMS collaboration, Search for narrow resonances decaying to dijets in pp collisions at $\sqrt{s}=13 \mathrm{TeV}$ using $12.9 \mathrm{fb}^{-1}$, CMS-PAS-EXO-16-032, CERN, Geneva Switzerland, (2016).

[20] L. Randall and R. Sundrum, A large mass hierarchy from a small extra dimension, Phys. Rev. Lett. 83 (1999) 3370 [hep-ph/9905221] [INSPIRE].

[21] T. Gherghetta and A. Pomarol, Bulk fields and supersymmetry in a slice of AdS, Nucl. Phys. B 586 (2000) 141 [hep-ph/0003129] [INSPIRE].

[22] Y. Grossman and M. Neubert, Neutrino masses and mixings in nonfactorizable geometry, Phys. Lett. B 474 (2000) 361 [hep-ph/9912408] [INSPIRE].

[23] M. Carena, A. Delgado, E. Ponton, T.M.P. Tait and C.E.M. Wagner, Warped fermions and precision tests, Phys. Rev. D 71 (2005) 015010 [hep-ph/0410344] [INSPIRE].

[24] M. Carena, E. Ponton, J. Santiago and C.E.M. Wagner, Electroweak constraints on warped models with custodial symmetry, Phys. Rev. D 76 (2007) 035006 [hep-ph/0701055] [INSPIRE].

[25] M. Baak et al., Updated status of the global electroweak fit and constraints on new physics, Eur. Phys. J. C 72 (2012) 2003 [arXiv:1107.0975] [InSPIRE].

[26] S.J. Huber and Q. Shafi, Fermion masses, mixings and proton decay in a Randall-Sundrum model, Phys. Lett. B 498 (2001) 256 [hep-ph/0010195] [INSPIRE].

[27] S.J. Huber, Flavor violation and warped geometry, Nucl. Phys. B 666 (2003) 269 [hep-ph/0303183] [INSPIRE].

[28] K. Agashe, A. Delgado, M.J. May and R. Sundrum, RS1, custodial isospin and precision tests, JHEP 08 (2003) 050 [hep-ph/0308036] [INSPIRE].

[29] G. Wojcik, J.L. Hewett and T.G. Rizzo, An alternative Randall-Sundrum model, to appear.

[30] C. Han, H.M. Lee, M. Park and V. Sanz, The diphoton resonance as a gravity mediator of dark matter, Phys. Lett. B 755 (2016) 371 [arXiv:1512.06376] [INSPIRE].

[31] H.M. Lee, M. Park and V. Sanz, Gravity-mediated (or composite) dark matter, Eur. Phys. J. C 74 (2014) 2715 [arXiv:1306.4107] [INSPIRE].

[32] H.M. Lee, M. Park and V. Sanz, Gravity-mediated (or composite) dark matter confronts astrophysical data, JHEP 05 (2014) 063 [arXiv:1401.5301] [INSPIRE].

[33] S. Kraml, U. Laa, K. Mawatari and K. Yamashita, Simplified dark matter models with a spin-2 mediator at the LHC, Eur. Phys. J. C 77 (2017) 326 [arXiv:1701.07008] [InSPIRE].

[34] J. Abdallah et al., Simplified models for dark matter searches at the LHC, Phys. Dark Univ. 9-10 (2015) 8 [arXiv: 1506.03116] [INSPIRE].

[35] J. Abdallah et al., Simplified models for dark matter and missing energy searches at the $L H C$, arXiv: 1409.2893 [INSPIRE].

[36] H. Georgi, A.K. Grant and G. Hailu, Brane couplings from bulk loops, Phys. Lett. B 506 (2001) 207 [hep-ph/0012379] [INSPIRE].

[37] H. Davoudiasl, J.L. Hewett and T.G. Rizzo, Brane localized curvature for warped gravitons, JHEP 08 (2003) 034 [hep-ph/0305086] [INSPIRE].

[38] H. Davoudiasl, J.L. Hewett and T.G. Rizzo, Brane localized kinetic terms in the Randall-Sundrum model, Phys. Rev. D 68 (2003) 045002 [hep-ph/0212279] [INSPIRE]. 
[39] H. Davoudiasl, J.L. Hewett and T.G. Rizzo, Experimental probes of localized gravity: on and off the wall, Phys. Rev. D 63 (2001) 075004 [hep-ph/0006041] [InSPIRE].

[40] H. Davoudiasl, J.L. Hewett and T.G. Rizzo, Bulk gauge fields in the Randall-Sundrum model, Phys. Lett. B 473 (2000) 43 [hep-ph/9911262] [INSPIRE].

[41] H. Davoudiasl, J.L. Hewett and T.G. Rizzo, Phenomenology of the Randall-Sundrum gauge hierarchy model, Phys. Rev. Lett. 84 (2000) 2080 [hep-ph/9909255] [INSPIRE].

[42] J.L. Hewett and T.G. Rizzo, $750 \mathrm{GeV}$ diphoton resonance in warped geometries, arXiv: 1603.08250 [INSPIRE].

[43] V. Silveira and A. Zee, Scalar phantoms, Phys. Lett. B 161 (1985) 136 [INSPIRE].

[44] S.I. Godunov, A.N. Rozanov, M.I. Vysotsky and E.V. Zhemchugov, Extending the Higgs sector: an extra singlet, Eur. Phys. J. C 76 (2016) 1 [arXiv:1503.01618] [INSPIRE].

[45] ATLAS collaboration, Search for a high-mass Higgs boson decaying to a $W$ boson pair in pp collisions at $\sqrt{s}=8 \mathrm{TeV}$ with the ATLAS detector, JHEP 01 (2016) 032 [arXiv: 1509.00389] [INSPIRE].

[46] B. Holdom, Two U(1)'s and $\epsilon$ charge shifts, Phys. Lett. B 166 (1986) 196 [InSPIRE].

[47] J. Alexander et al., Dark sectors 2016 workshop: community report, arXiv: 1608.08632 [INSPIRE].

[48] H. Davoudiasl and T.G. Rizzo, Bulk physics at a graviton factory, Phys. Lett. B 512 (2001) 100 [hep-ph/0104199] [INSPIRE].

[49] S. Dodelson, Modern cosmology, Academic Press, Amsterdam The Netherlands, (2003) [INSPIRE].

[50] P. Gondolo and G. Gelmini, Cosmic abundances of stable particles: improved analysis, Nucl. Phys. B 360 (1991) 145 [INSPIRE].

[51] P. Creminelli, A. Nicolis and R. Rattazzi, Holography and the electroweak phase transition, JHEP 03 (2002) 051 [hep-th/0107141] [INSPIRE].

[52] T. Konstandin and G. Servant, Cosmological consequences of nearly conformal dynamics at the TeV scale, JCAP 12 (2011) 009 [arXiv:1104.4791] [INSPIRE].

[53] T. Nihei, L. Roszkowski and R. Ruiz de Austri, Exact cross-sections for the neutralino WIMP pair annihilation, JHEP 03 (2002) 031 [hep-ph/0202009] [INSPIRE].

[54] T. Han, J.D. Lykken and R.-J. Zhang, On Kaluza-Klein states from large extra dimensions, Phys. Rev. D 59 (1999) 105006 [hep-ph/9811350] [InSPIRE].

[55] G.P. Lepage, A new algorithm for adaptive multidimensional integration, J. Comput. Phys. 27 (1978) 192 [INSPIRE].

[56] P.R. Kafle, S. Sharma, G.F. Lewis and J. Bland-Hawthorn, On the shoulders of giants: properties of the stellar halo and the milky way mass distribution, Astrophys. J. 794 (2014) 59 [arXiv: 1408.1787] [INSPIRE].

[57] A. Cuoco, M. Krämer and M. Korsmeier, Novel dark matter constraints from antiprotons in light of AMS-02, Phys. Rev. Lett. 118 (2017) 191102 [arXiv:1610.03071] [INSPIRE].

[58] M.-Y. Cui, Q. Yuan, Y.-L.S. Tsai and Y.-Z. Fan, Possible dark matter annihilation signal in the AMS-02 antiproton data, Phys. Rev. Lett. 118 (2017) 191101 [arXiv:1610.03840] [INSPIRE]. 
[59] AMS collaboration, M. Aguilar et al., Antiproton flux, antiproton-to-proton flux ratio and properties of elementary particle fluxes in primary cosmic rays measured with the Alpha Magnetic Spectrometer on the International Space Station, Phys. Rev. Lett. 117 (2016) 091103 [INSPIRE].

[60] A. Vittino and M. Di Mauro, A quantitative study of AMS-02 $e^{ \pm}$data. What can we learn about dark matter?, J. Phys. Conf. Ser. 718 (2016) 042060 [INSPIRE].

[61] AMS collaboration, M. Aguilar et al., Precision measurement of the $\left(e^{+}+e^{-}\right)$flux in primary cosmic rays from $0.5 \mathrm{GeV}$ to $1 \mathrm{TeV}$ with the Alpha Magnetic Spectrometer on the International Space Station, Phys. Rev. Lett. 113 (2014) 221102 [INSPIRE].

[62] AMS collaboration, M. Aguilar et al., Electron and positron fluxes in primary cosmic rays measured with the Alpha Magnetic Spectrometer on the International Space Station, Phys. Rev. Lett. 113 (2014) 121102 [INSPIRE].

[63] AMS collaboration, L. Accardo et al., High statistics measurement of the positron fraction in primary cosmic rays of 0.5-500 GeV with the Alpha Magnetic Spectrometer on the International Space Station, Phys. Rev. Lett. 113 (2014) 121101 [InSPIRE].

[64] L. Vecchi, WIMPs and un-naturalness, arXiv:1312.5695 [INSPIRE]. 